\title{
Diagnostic and Predictive Capabilities of the TCR Digital Platform
}

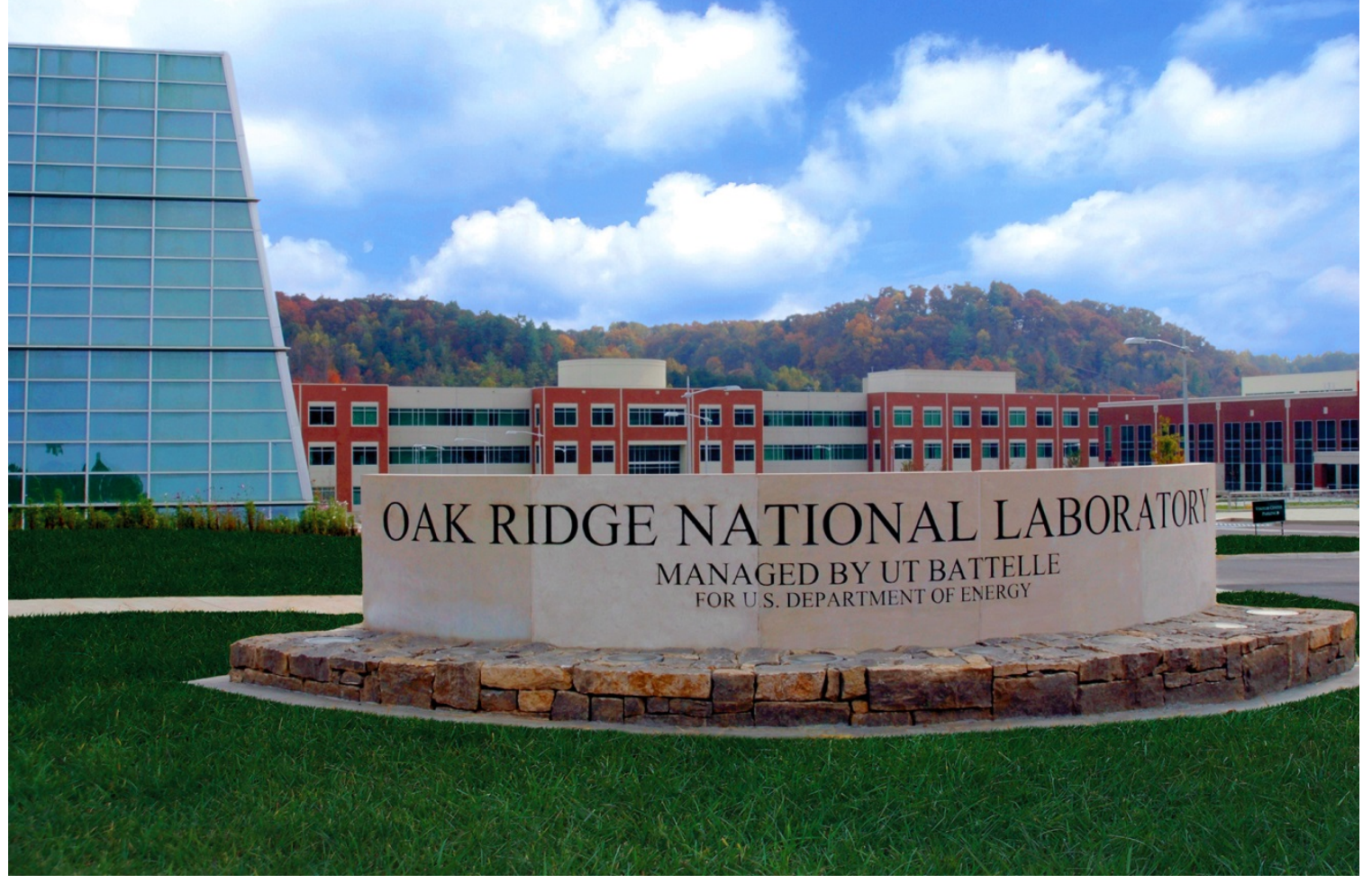

Luke Scime

Michael Sprayberry

David Collins

Alka Singh

Chase Joslin

Ryan Duncan

Joseph Simpson

Fred List III

Keith Carver

Alex Huning

James Haley

Vincent Paquit

September 2021

M2TC-21OR0404022 



\title{
DOCUMENT AVAILABILITY
}

Reports produced after January 1, 1996, are generally available free via US Department of Energy (DOE) SciTech Connect.

Website www.osti.gov

Reports produced before January 1, 1996, may be purchased by members of the public from the following source:

\author{
National Technical Information Service \\ 5285 Port Royal Road \\ Springfield, VA 22161 \\ Telephone 703-605-6000 (1-800-553-6847) \\ TDD 703-487-4639 \\ Fax 703-605-6900 \\ E-mail info@ntis.gov \\ Website http://classic.ntis.gov/
}

Reports are available to DOE employees, DOE contractors, Energy Technology Data Exchange representatives, and International Nuclear Information System representatives from the following source:

Office of Scientific and Technical Information

PO Box 62

Oak Ridge, TN 37831

Telephone 865-576-8401

Fax 865-576-5728

E-mail reports@osti.gov

Website https://www.osti.gov/

This report was prepared as an account of work sponsored by an agency of the United States Government. Neither the United States Government nor any agency thereof, nor any of their employees, makes any warranty, express or implied, or assumes any legal liability or responsibility for the accuracy, completeness, or usefulness of any information, apparatus, product, or process disclosed, or represents that its use would not infringe privately owned rights. Reference herein to any specific commercial product, process, or service by trade name, trademark, manufacturer, or otherwise, does not necessarily constitute or imply its endorsement, recommendation, or favoring by the United States Government or any agency thereof. The views and opinions of authors expressed herein do not necessarily state or reflect those of the United States Government or any agency thereof. 


\title{
DIAGNOSTIC AND PREDICTIVE CAPABILITIES OF THE TCR DIGITAL PLATFORM
}

\author{
Luke Scime \\ Michael Sprayberry \\ David Collins \\ Alka Singh \\ Chase Joslin \\ Ryan Duncan \\ Joseph Simpson \\ Fred List III \\ Keith Carver \\ Alex Huning \\ James Haley \\ Vincent Paquit
}

Date Published: September 2021

M2TC-21OR0404022

\author{
Prepared by \\ OAK RIDGE NATIONAL LABORATORY \\ Oak Ridge, TN 37831-6283 \\ managed by \\ UT-BATTELLE LLC \\ for the \\ US DEPARTMENT OF ENERGY \\ under contract DE-AC05-00OR22725
}





\section{CONTENTS}

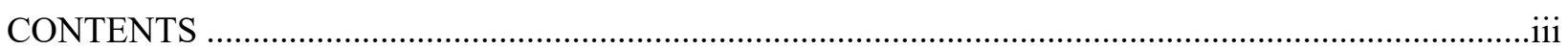

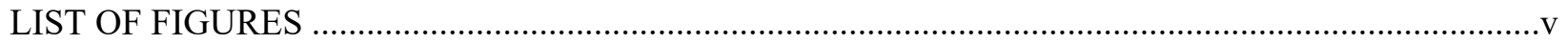

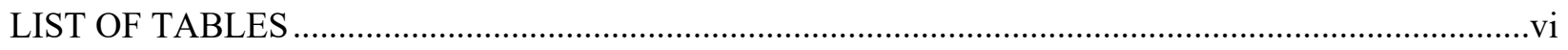

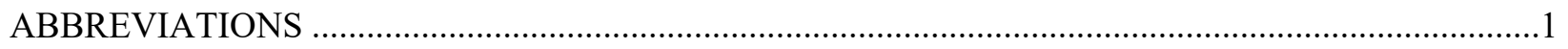

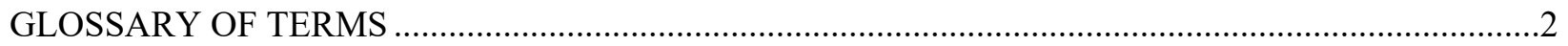

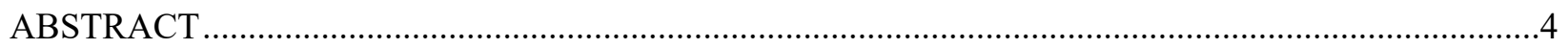

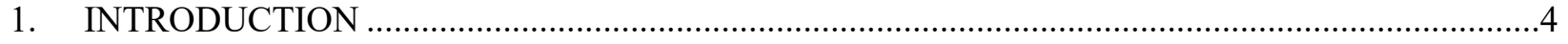

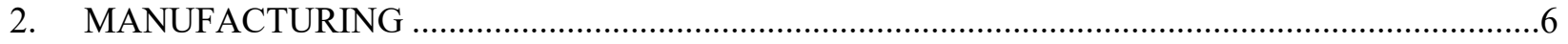

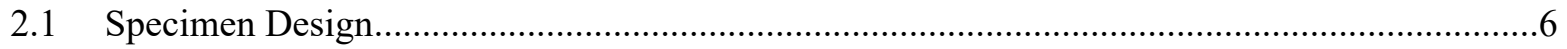

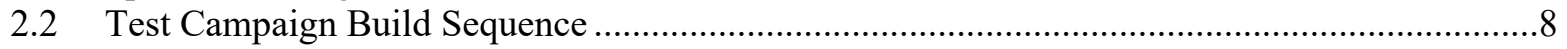

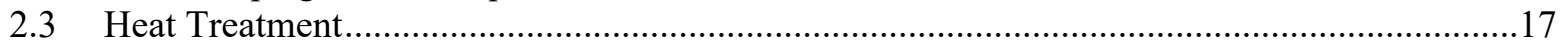

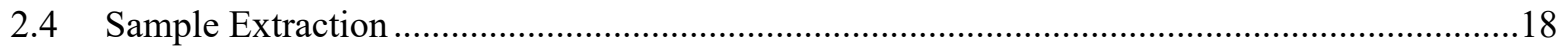

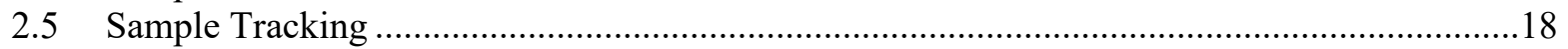

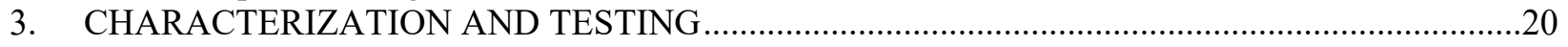

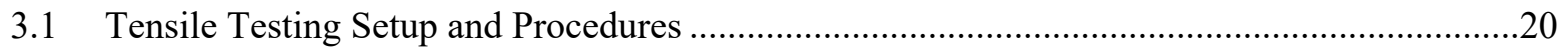

3.2 Tensile Testing Results and Observations ......................................................................22

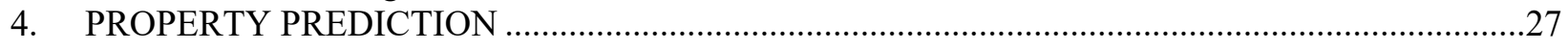

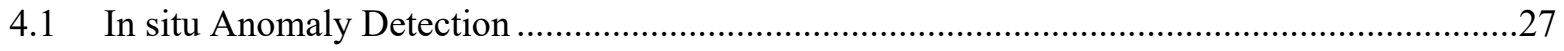

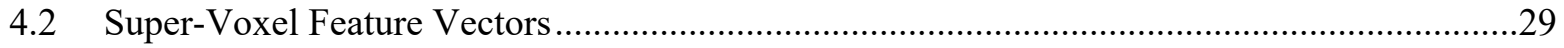

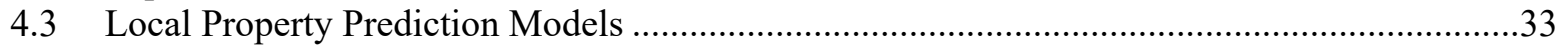

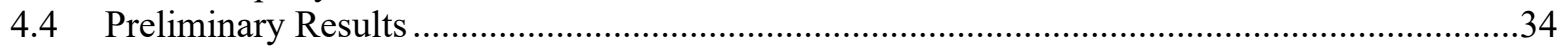

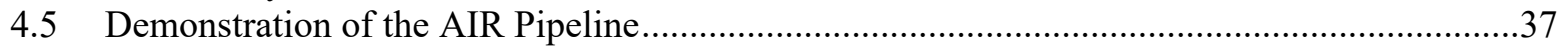

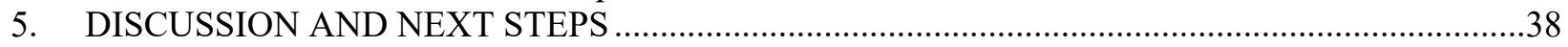

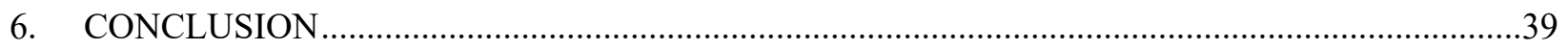

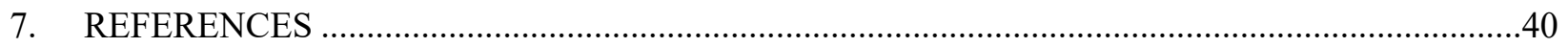




\section{LIST OF FIGURES}

Figure 1. A schematic representation of an example AIR [2] as implemented by the TCR program.

Figure 2. Examples of soot, recoater streaking, incomplete spreading, debris, swelling, and super-

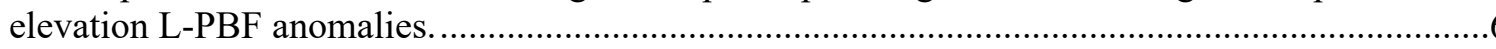

Figure 3. (Top) The four different as-built part geometries from which SS-J3 tensile samples can

be extracted. ......................................................................................................................

Figure 4. The build layout for "TCR Phase 1 Build 1" as viewed from the top with the Peregrine part identifiers overlaid.

Figure 5. CAD rendering of all the SS-J3 samples that could be extracted from "TCR Phase 1

Build 1.".

Figure 6. One in situ visible-light camera image of the powder bed captured during "TCR Phase 1

Build 1."

Figure 7. Isometric view of "TCR Phase 1 Build 1 " immediately after the printing operation.

Figure 8. The build layout for "TCR Phase 1 Build 2" as viewed from the top with the Peregrine part identifiers overlaid.

Figure 9. CAD rendering of all the SS-J3 samples that could be extracted from "TCR Phase 1 Build 2."

Figure 10. One in situ visible-light camera image of the powder bed captured during "TCR Phase 1 Build 2."

Figure 11. Isometric view of "TCR Phase 1 Build 2" immediately after the printing operation. . .12

Figure 12. The build layout for "TCR Phase 1 Build 3" as viewed from the top with the Peregrine part identifiers overlaid...

Figure 13. CAD rendering of all the SS-J3 samples that could be extracted from "TCR Phase 1 Build 3.".....

Figure 14. One in situ visible-light camera image of the powder bed captured during "TCR Phase 1 Build 3."

Figure 15. Isometric view of "TCR Phase 1 Build 3" immediately after the printing operation. ...............14

Figure 16. The build layout for "TCR Phase 1 Build 4" as viewed from the top with the Peregrine part identifiers overlaid...

Figure 17. CAD rendering of all the SS-J3 samples that could be extracted from "TCR Phase 1 Build 4."

Figure 18. One in situ visible-light camera image of the powder bed captured during "TCR Phase 1 Build 4 "

Figure 19. Isometric view of "TCR Phase 1 Build 4" immediately after the printing operation. ...............15

Figure 20. The build layout for "TCR Phase 1 Build 5" as viewed from the top with the Peregrine part identifiers overlaid.

Figure 21. CAD rendering of all the SS-J3 samples that could be extracted from "TCR Phase 1 Build 5."

Figure 22. One in situ visible-light camera image of the powder bed captured during "TCR Phase

1 Build 5."

Figure 23. Isometric view of "TCR Phase 1 Build 5" immediately after the printing operation.

Figure 24. A set of L-2 SS-J3 samples during extraction from an SSJ3-D geometry, still contained

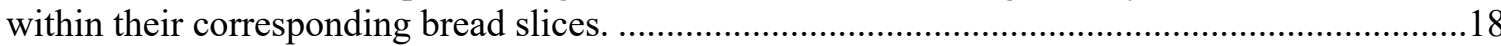

Figure 25. The DT web portal for instantiating a Concept Laser M2 L-PBF operation............................19

Figure 26. An example of DT search results for the Concept Laser M2 L-PBF operations......................20

Figure 27. Example AM SS 316L stress-strain curve with annotations highlighting the tensile properties of interest. 
Figure 28. Selected stress-strain curves from samples extracted from the SSJ3-C geometries printed in the "TCR Phase 1 Build 2" build.

Figure 29. Selected stress-strain curves for samples taken from SSJ3-C as-built parts printed using the BEST process parameter set in builds "TCR Phase 1 Build 2" and "TCR Phase 1 Build 3."

Figure 30. Selected stress-strain curves from "TCR Phase 1 Build 2" P24, featuring both interior tensile sample data and near-surface tensile sample data.

Figure 31. Selected stress-strain curves for "TCR Phase 1 Build 2" P24 and P27................................27

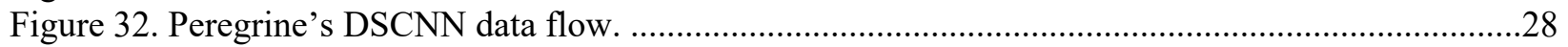

Figure 33. A graphical representation of the super-voxelization process..............................................31

Figure 34. Example super-voxel features from the "TCR Phase 1 Build 2" build overlaid on top of the corresponding in situ image of the powder bed.

Figure 35. Representation of the modified GNN with five hidden layers consisting of 32, 128, $128,128,128$, and 2 nodes, respectively, and an output layer. ......................................................

Figure 36. Predicted vs. ground truth UTS values for the four-feature LRM.........................................35

Figure 37. Predicted vs. ground truth UTS values for the 11 feature LRM.............................................36

Figure 38. Predicted vs. ground truth UTS values for the 11 feature GNN ..............................................37

Figure 39. (a) Preliminary UTS predictions at each super-voxel for the as-built parts in the "TCR Phase 1 Build 2" build overlaid on top of the corresponding in situ image of the powder bed.

\section{LIST OF TABLES}

Table 1. Description of Concept Laser M2 L-PBF builds performed for the TCR AIR test campaign.

Table 2. Chemical composition of powder Lot \#30 as reported by Praxair. ..............................................9

Table 3. Powder size distribution and flowability metrics of powder Lot \#30 as reported by Praxair.

Table 4. Laser process parameter sets used for the TCR test campaign builds. ......................................10

Table 5. Key process parameters held constant across the TCR test campaign builds................................10

Table 6. Furnace profile used for all TCR FY21 test campaign builds. ..................................................18

Table 7. Description of the Concept Laser M2 log file values used by the AIR .......................................30

Table 8. Description of super-voxel features available to the AIR to predict local tensile properties 31

Table 9. Confusion matrix for the SVM that uses a four-feature input vector. .........................................35

Table 10. Confusion matrix for the SVM that uses an 11 feature input vector. ..........................................35 


\section{ABBREVIATIONS}

$\begin{array}{ll}\text { AI } & \text { artificial intelligence } \\ \text { AIR } & \text { augmented intelligence relay } \\ \text { AM } & \text { additive manufacturing } \\ \text { API } & \text { application programming interface } \\ \text { CAD } & \text { computer aided design } \\ \text { CAM } & \text { computer aided machining } \\ \text { CSV } & \text { comma separated values [file] } \\ \text { DL } & \text { deep learning } \\ \text { DP } & \text { digital platform } \\ \text { DSCNN } & \text { dynamic segmentation convolutional neural network } \\ \text { EDM } & \text { electrical discharge machining } \\ \text { GNN } & \text { generalized neural network } \\ \text { LM } & \text { laser module } \\ \text { LoF } & \text { lack of fusion [porosity] } \\ \text { L-PBF } & \text { laser powder bed fusion } \\ \text { LRM } & \text { linear regression model } \\ \text { MDF } & \text { Manufacturing Demonstration Facility } \\ \text { ML } & \text { machine learning } \\ \text { OEM } & \text { original equipment manufacturer } \\ \text { ORNL } & \text { Oak Ridge National Laboratory } \\ \text { QR } & \text { quick response [code] } \\ \text { SS } & \text { stainless steel } \\ \text { SS 316L } & \text { stainless steel 316 low-carbon } \\ \text { STL } & \text { standard triangle language } \\ \text { SVM } & \text { support vector machine } \\ \text { TCR } & \text { Transformational Challenge Reactor } \\ \text { TE } & \text { total elongation } \\ \text { UE } & \text { uniform elongation } \\ \text { UTS } & \text { ultimate tensile strength } \\ \text { XCT } & \text { x-ray computed tomography } \\ \text { YTS } & \text { yield tensile strength } \\ & \end{array}$




\section{GLOSSARY OF TERMS}

The following key terms used throughout this report are defined here for disambiguation. Where possible, the additive manufacturing (AM) terminology used herein complies with ISO/ASTM 52900:2015 [1].

artificial intelligence (AI): an umbrella term referring to any computer algorithm that makes decisions intended to mimic those made by a human. The Transformational Challenge Reactor program considers raw data analyses to be performed by descriptive AIs, automated data summarization to be performed by diagnostic AIs, material property predictions to be made by predictive AIs, and autonomous component design decisions to be made by prescriptive AIs.

- $\quad$ supervised machine learning (ML): computer algorithms that learn to make decisions based on observation of labeled ground truth training data.

- unsupervised machine learning: computer algorithms that cluster or otherwise identify patterns in high-dimensional data without the need for labeled training data.

- deep learning (DL): ML algorithms that rely less strongly on human feature engineering to preprocess the data of interest but are generally more data intensive to implement.

- heuristic: a human-engineered decision-making rule that can be implemented by a computer.

augmented intelligence relay (AIR): a sequence of AI algorithms that ingest raw process data and predict relevant component properties. At each interface in the relay, human domain experts observe the intermediate results and perform feature-engineering to better structure the data. The relay approach is designed to reduce the number of expensive characterization tests that must be performed to achieve the goals of a predictive AI.

database: the collection of metadata that comprise the digital threads for all the components manufactured at the Manufacturing Demonstration Facility (MDF). Strictly, the heavier process data (e.g., in situ images) are not stored in the database; they are stored within the designated file systems, and the database only moderates and facilitates data upload and retrieval by using application programing interfaces.

Digital Platform (DP): the cyberphysical infrastructure under development at the MDF that is enabling novel design and qualification paradigms for advanced manufacturing.

digital thread: the totality of the design intent information, data, and metadata collected during the fabrication of a component. The digital thread enables the instantiation of a corresponding digital twin model. Ultimately, digital threads are ingested by the AIR to predict relevant component properties based on collected process data.

Digital Tool (DT): a web-based client developed at the MDF to facilitate metadata collection for the different operations with the goal of creating a digital thread for each manufactured component. This application is the primary interface between most users and the DP database.

digital twin: a computer representation and model of a real object, assembly, or system. This model is updated based on data collected from its physical twin; this contrasts with a computer model that relies solely on aggregated or representative data from many identical components.

digital workflow: the interaction between the physical manufacturing process, human technicians, and the DP. 
keyhole porosity: forms when the base of the keyhole vapor depression collapses or "pinches off," trapping a pore when the melt pool solidifies. These porosities are generally spherical. Higher energy density laser parameters, particularly at lower scan speeds, can increase the likelihood of this porosity occurring.

lack of fusion porosity: caused when adjacent melt pool tracks do not fully overlap. These porosities are generally irregularly shaped and often have unfused powder particles inside the pores. Lower energy density laser parameters can increase the likelihood of this porosity occurring.

operation: to facilitate scaling the digital workflows, each manufacturing process is decomposed into a sequence of operations. A digital thread is then merely a list of all operations that were performed to fabricate the component along with links to the associated data and metadata.

part: any component manufactured at the MDF. Any as-built additively manufactured part can be separated into child parts, referred to as samples.

software tool: any custom or commercial software used to facilitate a digital workflow or specific operation.

- Peregrine: a software tool designed to provide a comprehensive suite of data collection, analysis, and visualization capabilities for powder bed AM systems. 


\begin{abstract}
The Transformational Challenge Reactor program is leveraging additive manufacturing (AM) technologies to fabricate the nuclear components required to assemble a microreactor core. Compared with traditional manufacturing processes, AM allows the interior of the component to be directly observed during manufacturing. This unique capability promises significant possibilities for creating a new paradigm for nuclear component qualification by leveraging in situ process data. This report describes the FY21 efforts to predict material tensile properties based on data collected during the laser powder bed fusion printing process. The primary focus of this report is the test campaign designed to generate the large quantities of training data required to implement artificial intelligence algorithms that can predict these material properties. Preliminary prediction results and a demonstration of the overall data collection, analysis, and visualization pipeline are also provided.
\end{abstract}

\title{
1. INTRODUCTION
}

In FY21, the Transformational Challenge Reactor (TCR) team began implementing a new rapid component qualification paradigm for stainless steel 316L (SS 316L) parts manufactured using the Concept Laser M2 laser powder bed fusion (L-PBF) additive manufacturing (AM) process. In this new paradigm, critical components will be certified based on "instance-specific" data as opposed to a more traditional reliance on data aggregated from the characterization and historical performance of many identical components. To enable this approach, visible-light images of the powder bed and various machine health sensor streams are collected in situ during the L-PBF printing operation. These sensors are described in a previously released TCR report by Scime et al. [2]. The data and metadata from the printing operation, as well several postprocessing and characterization operations, are collected and stored using the Manufacturing Demonstration Facility's (MDF) cyberphysical Digital Platform (DP) infrastructure. Additional details about the DP can be found in Scime et al. [3], and the relevant terminology is defined in the glossary of this report.

Because the in situ data are very high dimensional in nature and not easily interpretable by humans or physics-based models, it is proposed that modern artificial intelligence (AI) algorithms are required to achieve the desired component qualification paradigm. The existential challenge of predicting part properties using AI is the collection of large quantities of training data with associated ground truth labels. In the case of the TCR program, the primary ground truth labels are the tensile properties as measured by using tensile load frames. Taking as input the sensor and metadata from a part's digital thread, which is the sequence of operations used to manufacture that part, a naive approach in which one AI is used to directly predict part performance (i.e., "will the part fail?") would require at least $10^{9}$ tensile specimens. Additionally, such a schema should be expected to generalize poorly to different component geometries and loading conditions. Because the direct approach is wholly infeasible, the TCR program is leveraging the novel concept of an augmented intelligence relay (AIR) [3] to reduce the ground truth data requirement to approximately $10^{4}$ tensile specimens and improve the generalizability of the final predictive models. The TCR program will be the first known demonstration of an AIR, or similar schema, in the AM domain.

In an AIR (Figure 1), a sequence of targeted AI algorithms is used to solve subparts of the overall problem. At the beginning of the AIR, ground truth data are relatively easy to collect, and data-intensive deep learning (DL) AI algorithms can be used. As the data flow through the AIR, the ground truth data become more expensive to collect, and less data-intensive models must be used. For the TCR AIR specifically, in situ image stacks are first analyzed for anomalies using a pixel-wise DL segmentation algorithm, which trains using $10^{8}$ annotated pixels. Then, human-engineered feature vectors that summarize the anomaly detections and incorporate the printer health data are constructed at a super-voxel 
level. These super-voxels are also designed to mitigate edge effects and reduce the data volume. Next, localized tensile properties are predicted using a machine learning (ML) algorithm, which ingests the feature vectors and trains using $10^{4}$ tensile test results. Finally, the localized material property predictions can be fed into a physics-based model of component performance, which only requires sufficient data on the order of $10^{2}$ test artifacts for validation.

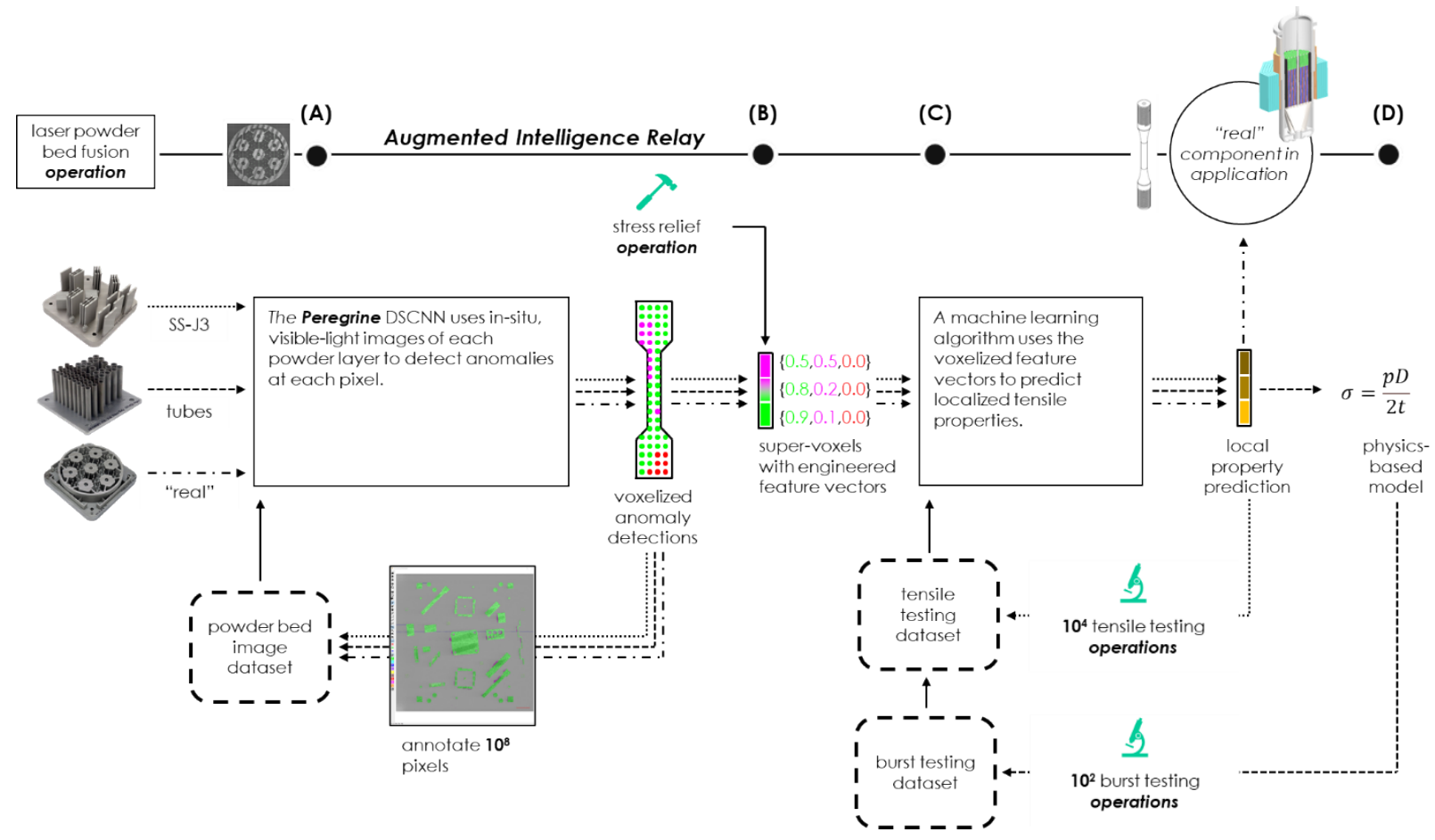

Figure 1. A schematic representation of an example AIR [2] as implemented by the TCR program. The AI algorithms start by leveraging large quantities of cheap ground truth training data, and as the data flow moves from left to right, the AI algorithms become less data intensive. Expert human domain knowledge is injected at each interface between the AI algorithms to facilitate the data flow and further reduce the training data requirements.

Several surface-visible in situ process anomalies common in L-PBF processes are referred to throughout this report. These include soot, recoater streaking, incomplete spreading, debris, swelling, and superelevation, which are defined in Scime et al. [3]. Examples of each of these anomalies are shown in Figure 2 . 


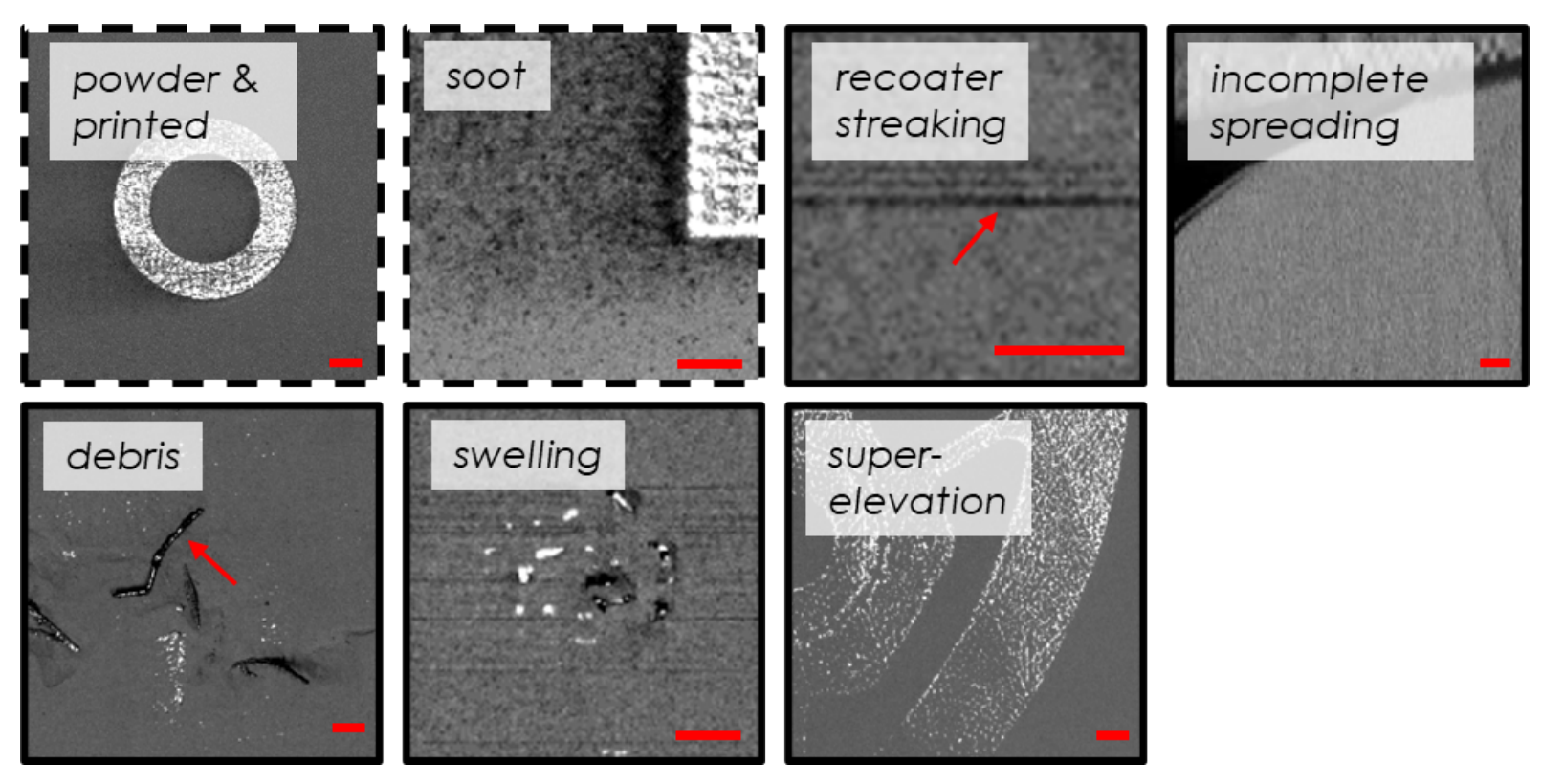

Figure 2. Examples of soot, recoater streaking, incomplete spreading, debris, swelling, and super-elevation

L-PBF anomalies. An example of nominally printed material surround by anomaly-free powder is also provided. The scale bars are $5 \mathrm{~mm}$ in length. In situ images marked with a dashed border were captured after layer fusion, and images with a solid border were captured after powder spreading.

This report focuses primarily on steps two and three of the TCR AIR. Some information about the descriptive AI in the first step is provided for context; however, for detailed information, readers should refer to Scime et al. [3]. The fourth step of the AIR is addressed in Huning et al. [4]. Section 2 discusses the manufacture of the tensile specimens and test artifacts. Section 3 describes the tensile testing conditions and reports selected characterization results. Section 4 describes the algorithms within the AIR in detail and presents preliminary material property predictions. Finally, progress made during FY21 and work proposed for FY22 are discussed.

\section{MANUFACTURING}

\subsection{SPECIMEN DESIGN}

For the AIR to be effective, it is crucial for the ground truth tensile data to be highly localized and easily generalizable to the TCR reactor core geometries. The resolution of the ground truth data is effectively the volume of the tensile specimen's gauge section. The smaller this volume is, the easier it is for the AIR to correlate measured tensile properties with the L-PBF anomaly detections, which have a resolution on the order of $0.1 \mathrm{~mm}$. Additionally, because many of the TCR reactor core geometries consist of thin-wall structures with thicknesses on the order of $1 \mathrm{~mm}$ or less, the AIR must be exposed to example ground truth data collected from thin wall structures. In opposition, it is difficult to consistently print high-aspect ratio structures thinner than $0.5 \mathrm{~mm}$ on the Concept Laser M2. This combination of constraints drove the decision to select the SS-J3 tensile specimen geometry with a gauge volume of $5.0 \mathrm{~mm} \times 1.2 \mathrm{~mm} \times 0.75$ $\mathrm{mm}$ for use throughout the FY21 test campaign. To preserve compatibility with the TCR program's irradiation efforts, the nominal SS-J3 dimensions are based on Oak Ridge National Laboratory's (ORNL’s) Generic Metal Irradiation Specimen standard [5]. 
The decision was also made to print larger parts and then extract the SS-J3 samples using wire electrical discharge machining (EDM), as opposed to directly printing the SS-J3s, for two reasons. First, printing free-standing or unsupported SS-J3s within the Concept Laser M2's build volume is generally impossible. Second, it is important to expose the AIR to ground truth data collected from variously sized component geometries to improve generalizability and capture the known influence of local geometry on the parts' thermal histories. Therefore, tensile samples were extracted from four different types of part geometries: a nominally $750 \mu \mathrm{m}$ thick wall (SSJ3-A), a nominally $1.5 \mathrm{~mm}$ thick wall (SSJ3-B), a nominally $5.0 \mathrm{~mm}$ thick wall (SSJ3-C), and an approximately $40 \mathrm{~mm}$ cube (SSJ3-D). Each as-built part can respectively source up to 24, 24, 200, or 576 tensile samples. The samples are located at four different levels in the build height designated L-1, L-2, L-3, and L-4 with each level nominally spaced apart by $19 \mathrm{~mm}$.

Computer aided design (CAD) renderings of each as-built part geometry and its associated samples are shown in Figure 3.
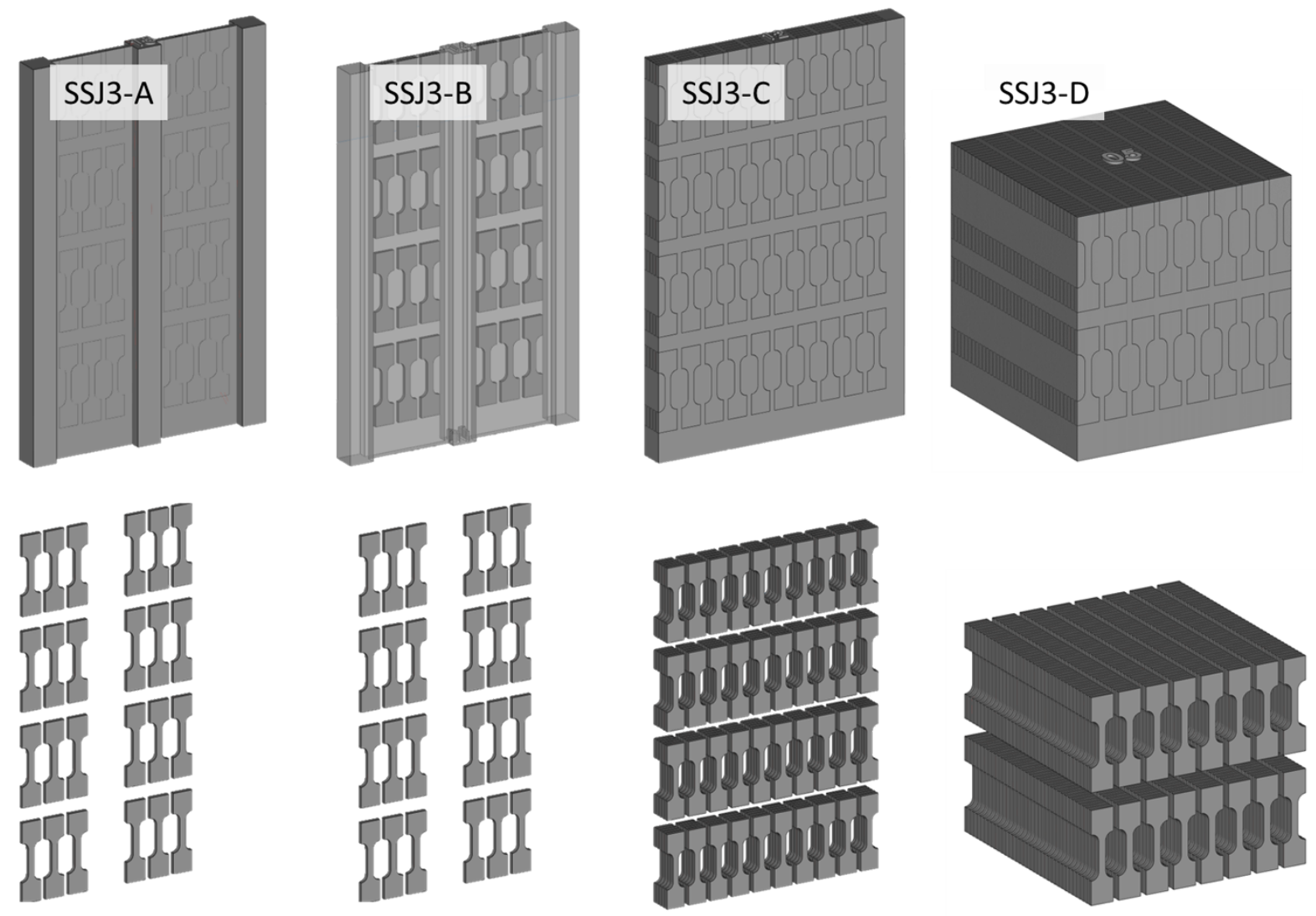

Figure 3. (Top) The four different as-built part geometries from which SS-J3 tensile samples can be extracted. (Bottom) The associated SS-J3 samples for each as-built part geometry.

Although not currently used by the AIR, each test campaign build was also printed with MDF-standard powder sampling containers and x-ray computed tomography (XCT) specimens. Burst tubes were also printed with each test campaign build. The burst tube geometries are described in Huning et al. [4] and will be used to validate the final AIR predictions in FY22. 


\subsection{TEST CAMPAIGN BUILD SEQUENCE}

To produce the ground truth tensile property training data for the AIR, a sequence of Concept Laser M2 L-PBF builds were designed and printed during FY21. This sequence is referred to throughout this report as the test campaign and consists of calibration builds, builds to "debug" the digital workflow and associated procedures, and builds designed to produce large quantities of SS-J3 samples and burst tubes under different experimental conditions. The printer coordinate system used in this report is defined in Scime et al. [6] and designates the vertical build direction as the $+z$-axis, the printer-front to printer-back direction as the $+y$-axis, and the printer-left to printer-right direction as the $+x$-axis. Table 1 enumerates all the builds undertaken as part of the test campaign. The remainder of this section focuses on the five builds that were designed to introduce specific types of anomalies and defects into the printed components so that the collected in situ data can be successfully correlated to the tensile properties using the AIR.

Table 1. Description of Concept Laser M2 L-PBF builds performed for the TCR AIR test campaign.

\begin{tabular}{|c|c|c|}
\hline Build date & Build name & Description \\
\hline $2020-11-18$ & M2 Calibration Plate & Used for in situ camera calibration. \\
\hline 2020-11-19 & TCR Phase 0 Build 1 & $\begin{array}{l}\text { Used to develop the SS-J3 tensile sample extraction, } \\
\text { tracking, and tensile testing procedures and digital workflow. } \\
\text { Tensile samples for the TCR irradiation thrust were also } \\
\text { extracted from this build. }\end{array}$ \\
\hline $2020-12-02$ & M2 Calibration Build 2 & Used for in situ camera calibration. \\
\hline 2020-12-04 & TCR Phase 0 HT Test & $\begin{array}{l}\text { Used to test the heat-treatment procedures and develop the } \\
\text { optimal furnace temperature profile. }\end{array}$ \\
\hline $2020-12-29$ & Swagelock Test & $\begin{array}{l}\text { Used to quantify the dimensional tolerances of the burst } \\
\text { tubes to ensure successful interfaces with the ex situ testing } \\
\text { equipment. }\end{array}$ \\
\hline \multicolumn{2}{|c|}{ January 2021-March 2021} & TCR stop work period. \\
\hline 2021-04-12 & M2 Calibration Plate 3 & Used for in situ camera calibration. \\
\hline 2021-04-14 & M2 Calibration Plate 4 & Used for in situ camera calibration. \\
\hline 2021-04-16 & TCR Phase 1 Build 2 & $\begin{array}{l}\text { This build contained four clusters of SS-J3 bulk parts and } \\
\text { burst tubes with each cluster printed with different laser } \\
\text { processing parameters. Up to } 2,000 \text { SS-J3 samples and } 16 \\
\text { burst tubes are targeted for extraction from this build. }\end{array}$ \\
\hline 2021-04-28 & TCR Phase 1 Build 3 & $\begin{array}{l}\text { This build contained SS-J3 bulk parts and burst tubes printed } \\
\text { at an angle relative to the z-axis (build direction). Different } \\
\text { parts were built with different laser modules (LMs). Up to } \\
1,000 \text { SS-J3 samples and } 12 \text { burst tubes are targeted for } \\
\text { extraction from this build. }\end{array}$ \\
\hline \multicolumn{2}{|c|}{ May 2021-June 2021} & Manufacturing TCR core demonstration components. \\
\hline 2021-06-30 & TCR Phase 1 Build 1 & This build failed to print successfully. \\
\hline 2021-07-13 & TCR Phase 1 Build 1 & $\begin{array}{l}\text { This build contained four clusters of SS-J3 bulk parts and } \\
\text { burst tubes with each cluster built with "good" laser } \\
\text { processing parameters. Different parts were built with } \\
\text { different LMs. Up to } 500 \text { SS-J3 samples and } 16 \text { burst tubes } \\
\text { are targeted for extraction from this build. }\end{array}$ \\
\hline 2021-07-20 & TCR Soot Dump Experiment & $\begin{array}{l}\text { Used to design optimal laser processing parameters to induce } \\
\text { soot deposition. }\end{array}$ \\
\hline
\end{tabular}




\begin{tabular}{c|c|l} 
Build date & Build name & Description \\
\hline 2021-08-03 & TCR Phase 1 Build 4 & $\begin{array}{l}\text { This build contained four clusters of SS-J3 bulk parts and } \\
\text { burst tubes with the clusters exposed to excessive soot } \\
\text { deposition sourced by parts located upstream of the argon } \\
\text { crossflow. Different parts were built with different LMs. Up } \\
\text { to 1,000 SS-J3 samples and 16 burst tubes are targeted for } \\
\text { extraction from this build. }\end{array}$ \\
\hline \multirow{2}{*}{ 2021-08-23 } & TCR Phase 1 Build 5 & $\begin{array}{l}\text { This build contained four clusters of SS-J3 bulk parts and } \\
\text { burst tubes with several clusters exposed to intentionally } \\
\text { generated recoater streaking and incomplete spreading } \\
\text { anomalies. Different parts were built with different LMs. Up } \\
\text { to 1,000 SS-J3 samples and 16 burst tubes are targeted for } \\
\text { extraction from this build. }\end{array}$
\end{tabular}

Throughout the five builds listed in Table 1 with bold font, all SS-J3 samples and burst tubes were printed using one of the laser process parameter sets identified in Table 2. Where the NOMINAL parameter set is provided by Concept Laser, the BEST parameter set is designed to minimize porosity, the lack-of-fusion (LOF) parameter set is designed to induce LoF porosity, the KEYHOLE parameter set is designed to induce keyholing porosity, and the SOOT parameter set is designed to intentionally generate large quantities of soot. The LOF and KEYHOLE parameter sets are respectively lower and higher energy densities than the NOMINAL parameter set and are also expected to result in different as-printed microstructures. The Concept Laser M2 printer has two LMs, both of which were used during the test campaign as noted in the description of each build, provided below. The gas flow rate and powder dosing parameters were manually adjusted during several of the builds to intentionally induce anomalies, and these changes are described along with each relevant build. Key processing parameters that remained fixed throughout these builds are reported in Table 3. All five builds were printed with SS 316L powder from Praxair and sourced from Lot \#30. Table 4 specifies the chemical composition of the powder lot as reported by the manufacturer. Table 5 specifies the powder size distribution and flowability metrics. The builds were performed in accordance with TCR-AM-PLAN-002, TCR Digital Platform AM Fabrication and Test Plan [7], and TCR-AM-PLAN-003, TCR Regulatory AM Fabrication and Burst Test Plan [8].

Table 2. Laser process parameter sets used for the TCR test campaign builds.

\begin{tabular}{c|c|c|c|c|c|c}
$\begin{array}{c}\text { Parameter } \\
\text { set }\end{array}$ & $\begin{array}{c}\text { Laser beam } \\
\text { power }(\mathbf{W})\end{array}$ & $\begin{array}{c}\text { Laser beam } \\
\text { speed }(\mathbf{m m} / \mathbf{s})\end{array}$ & $\begin{array}{c}\text { Hatch } \\
\text { spacing } \\
(\boldsymbol{\mu m})\end{array}$ & $\begin{array}{c}\text { Nominal } \\
\text { laser spot } \\
\text { size }(\boldsymbol{\mu m})\end{array}$ & $\begin{array}{c}\text { Stripe width } \\
\mathbf{( m m )}\end{array}$ & $\begin{array}{c}\text { Scan rotation } \\
\text { (degrees/layer) }\end{array}$ \\
\hline NOMINAL & 370 & 1,350 & 90 & 130 & 10 & 67 \\
BEST & 380 & 800 & 110 & 125 & 18 & 67 \\
LOF & 290 & 1,200 & 150 & 50 & 18 & 67 \\
KEYHOLE & 290 & 800 & 70 & 125 & 18 & 67 \\
SOOT & 290 & 1,200 & 70 & 50 & 18 & 90
\end{tabular}

Table 3. Key process parameters held constant across the TCR test campaign builds.

\begin{tabular}{c|c} 
Parameter & Value \\
\hline Nominal layer thickness $(\mu \mathrm{m})$ & 50 \\
Preheat temperature $\left({ }^{\circ} \mathrm{C}\right)$ & Room temperature \\
Skywriting parameters & OEM standard \\
Shielding gas & argon
\end{tabular}

Table 4. Chemical composition of powder Lot \#30 as reported by Praxair. All values are in weight percent.

\begin{tabular}{|c|c|c|c|c|c|c|c|c|c|c|c|c|c|}
\hline $\mathbf{C}$ & $\mathbf{C o}$ & $\mathbf{C r}$ & $\mathbf{C u}$ & $\mathbf{F e}$ & $\mathbf{M n}$ & $\mathbf{M o}$ & $\mathbf{N}$ & $\mathbf{N i}$ & $\mathbf{O}$ & $\mathbf{P}$ & $\mathbf{S}$ & $\mathbf{S i}$ & Other \\
\hline$<0.005$ & 0.08 & 17.01 & 0.00 & Bal & 1.29 & 2.48 & 0.01 & 12.67 & 0.03 & $<0.005$ & 0.005 & 0.59 & $<0.1$ \\
\hline
\end{tabular}


Table 5. Powder size distribution and flowability metrics of powder Lot \#30 as reported by Praxair.

\begin{tabular}{c|c} 
Parameter & Value \\
\hline D10 $(\mu \mathrm{m})$ & 20 \\
D50 $(\mu \mathrm{m})$ & 31 \\
D90 $(\mu \mathrm{m})$ & 42.5 \\
Apparent density $\left(\mathrm{g} / \mathrm{cm}^{3}\right)$ & 4.12 \\
Hall flow test $(\mathrm{s})$ & 16
\end{tabular}

Build 2021-07-13, “TCR Phase 1 Build 1," was designed to collect baseline material property data from components manufactured under nominal printing conditions. This build contained 39 parts (Figure 4) from which a maximum of 3,296 SS-J3 samples could be extracted (Figure 5). Sixteen burst tubes, two powder sampling containers, four XCT specimens, and one thermocouple well (Section 2.3) were also printed. The SS-J3 samples were sourced from four clusters of as-printed parts, and each cluster comprised one each of the SSJ3-A, SSJ3-B, SSJ3-C, and SSJ3-D part geometries. The burst tubes and XCT specimens were evenly distributed among the clusters. All parts that contained SS-J3 samples and burst tubes were printed with contours and using the NOMINAL process parameters with an offset-tocontour value of $110 \mu \mathrm{m}$. Two clusters were printed with LM-1, and two clusters were printed with LM2. Variations in the in situ sensor signatures, process anomalies, and tensile properties are expected because of (1) differing thermal conditions across the part geometries, (2) the LM used for melting, (3) the spatial location of the samples across the build area, and (4) the spatial location of the samples in the vertical build direction. Figure 6 shows a representative in situ image from one layer of the build, and Figure 7 shows the completed build before the heat-treatment operation.

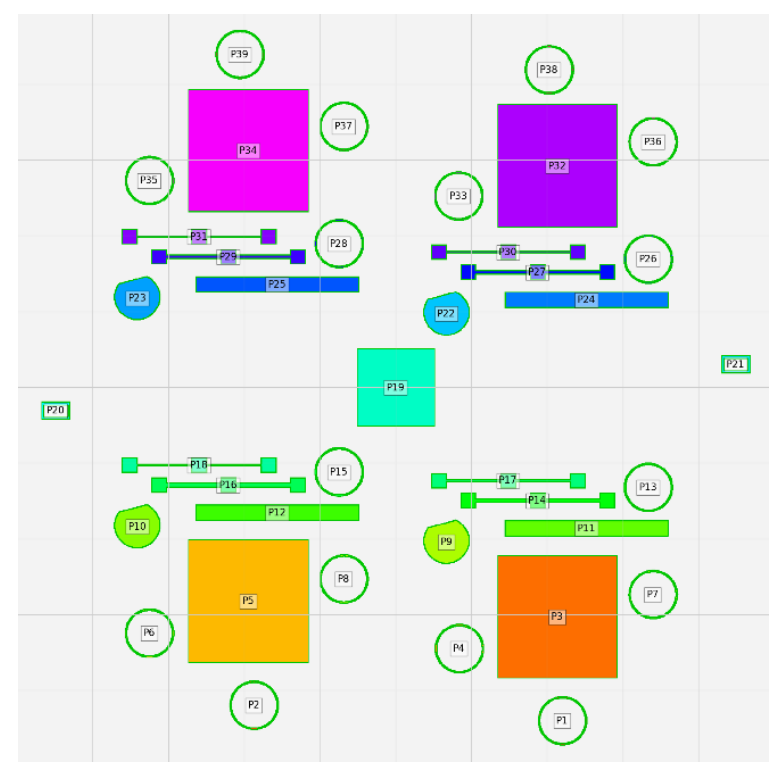

Figure 4. The build layout for "TCR Phase 1 Build 1" as viewed from the top with the Peregrine part identifiers overlaid.

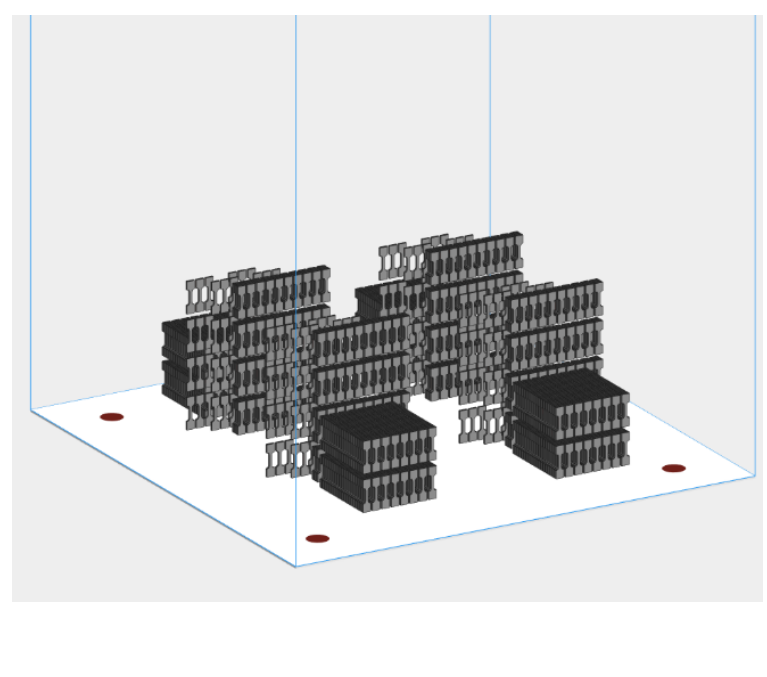

Figure 5. CAD rendering of all the SS-J3 samples that could be extracted from "TCR Phase 1 Build 1." 


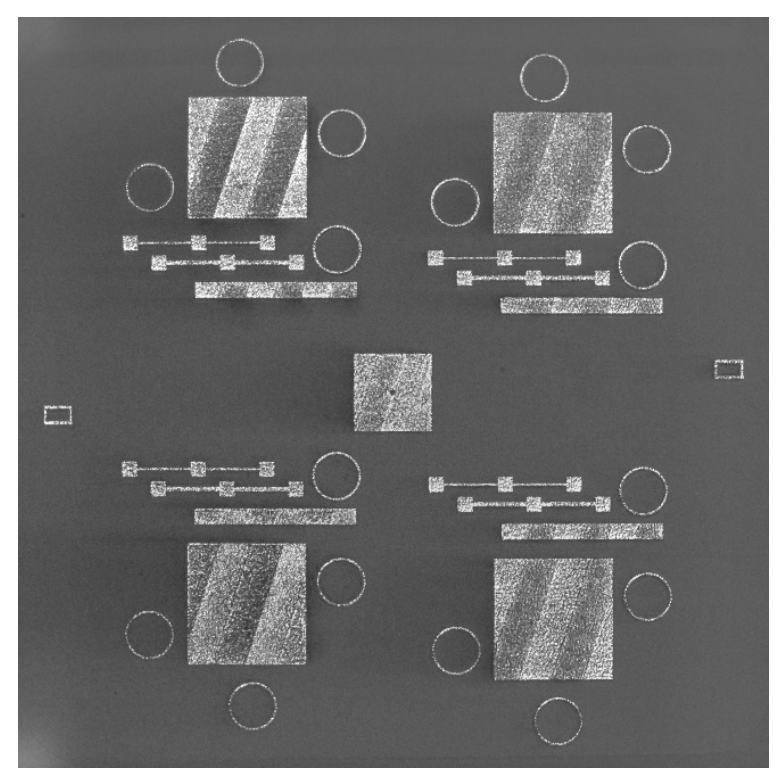

Figure 6. One in situ visible-light camera image of the powder bed captured during "TCR Phase 1 Build 1."

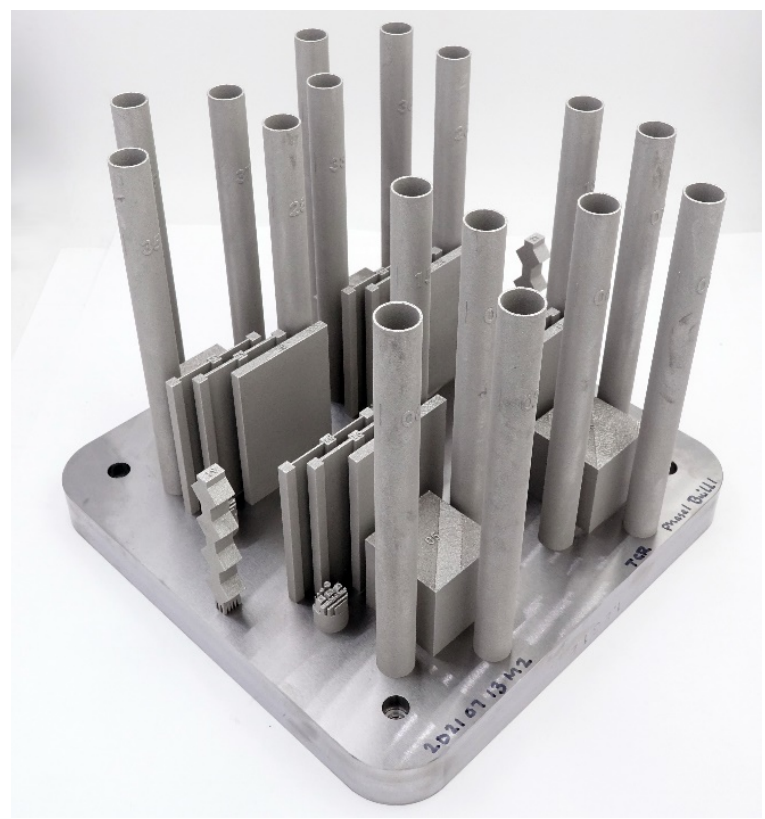

Figure 7. Isometric view of "TCR Phase 1 Build 1" immediately after the printing operation.

Build 2021-04-16, "TCR Phase 1 Build 2," was designed to collect data on samples with an extremely wide spectrum of material properties, essentially serving as expected component performance bounds for the test campaign. This build contained 39 parts (Figure 8) from which a maximum of 3,296 SS-J3 samples could be extracted (Figure 9). Sixteen burst tubes, two powder sampling containers, four XCT specimens, and one thermocouple well were also printed. The SS-J3 samples were sourced from four clusters of as-printed parts, and each cluster comprised one each of the SSJ3-A, SSJ3-B, SSJ3-C, and SSJ3-D part geometries. The burst tubes and XCT specimens were evenly distributed among the clusters. All the clusters were printed with LM-1, and each cluster was assigned either the NOMINAL, BEST, LOF, or KEYHOLE parameter set. All clusters were printed without contours, and all clusters used an offset-to-contour value of $1 \mu \mathrm{m}$. Variations in the in situ sensor signatures, process anomalies, and tensile properties are expected because of (1) differing thermal conditions across the part geometries, (2) the spatial location of the samples across the build area, (3) the spatial location of the samples in the vertical build direction, (4) the porosity content resulting from differing laser process parameters, and (5) the asbuilt microstructure resulting from differing laser process parameters. Figure 10 shows a representative in situ image from one layer of the build, and Figure 11 shows the completed build before the heat-treatment operation. 


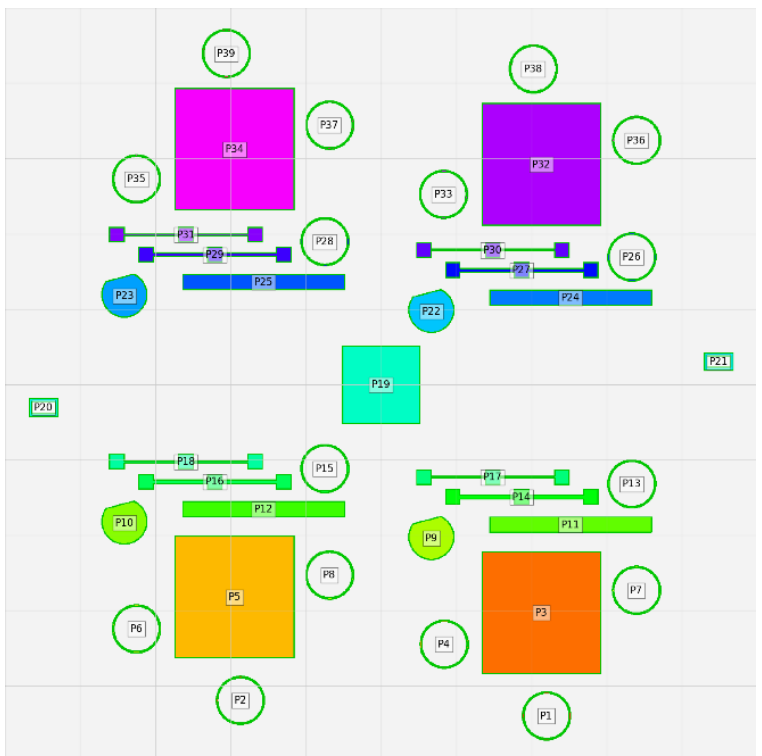

Figure 8. The build layout for "TCR Phase 1 Build 2" as viewed from the top with the Peregrine part identifiers overlaid.

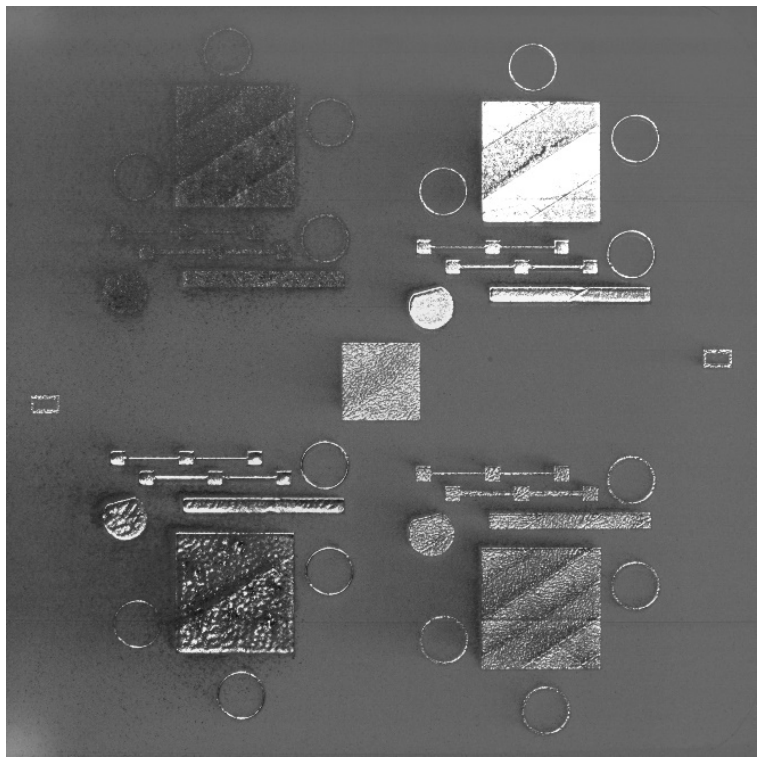

Figure 10. One in situ visible-light camera image of the powder bed captured during "TCR Phase 1 Build 2."

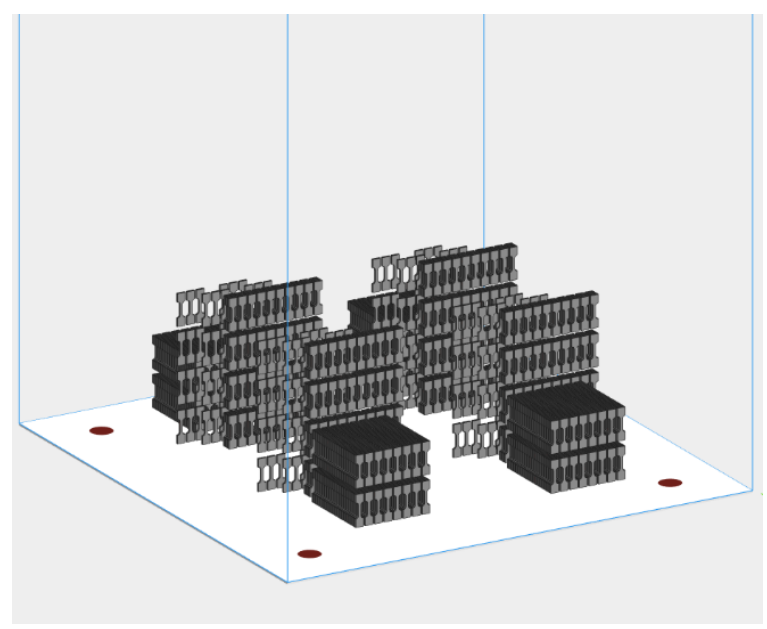

Figure 9. CAD rendering of all the SS-J3 samples that could be extracted from "TCR Phase 1 Build 2."

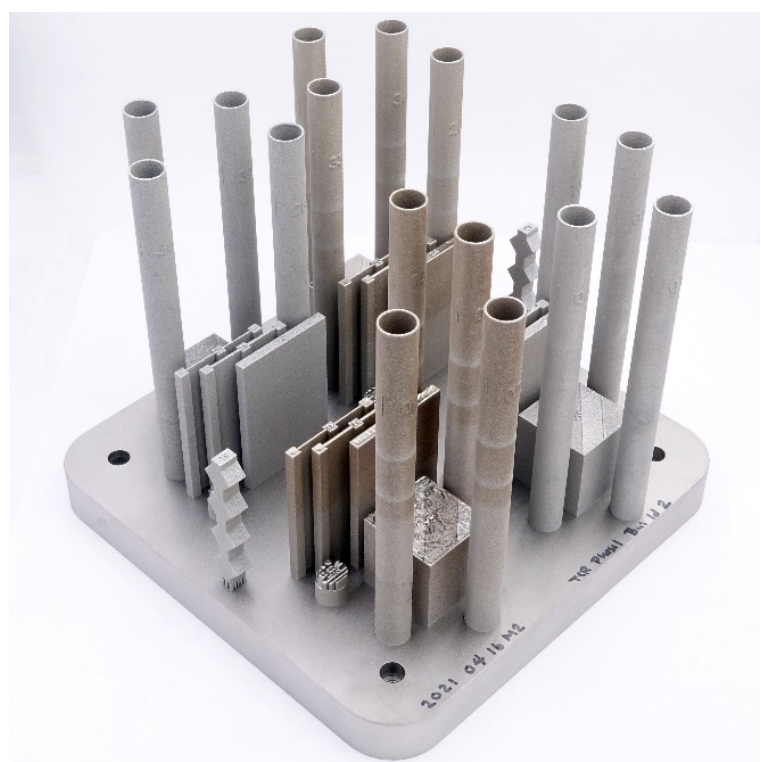

Figure 11. Isometric view of "TCR Phase 1 Build 2" immediately after the printing operation.

Build 2021-04-28, “TCR Phase 1 Build 3," was designed to collect data on components built with significant overhangs. This build contained 22 parts (Figure 12) from which a maximum of 896 SS-J3 samples could be extracted (Figure 13). Twelve burst tubes and two powder sampling containers were also printed. Because of space constraints on the build plate, the thermocouple well from a previous build was reused during heat treatment instead of printing a new one. The SS-J3 samples were sourced from 
four clusters of as-printed parts, and each cluster comprised one each of the SSJ3-A and SSJ3-C part geometries. The burst tubes were evenly distributed among the clusters. The SSJ3-A, SSJ3-C, and burst tube parts were all inclined at a $30^{\circ}$ angle relative to the vertical build direction. One cluster of SS-J3 samples and burst tubes was printed using the NOMINAL process parameters and LM-1, one cluster was printed using the NOMINAL process parameters and LM-2, one cluster was printed using the BEST process parameters and LM-1, and one cluster was printed using the BEST process parameters and LM-2. All clusters were printed with the contour process parameters provided by Concept Laser with an offsetto-contour value of $110 \mu \mathrm{m}$. Variations in the in situ sensor signatures, process anomalies, and tensile properties are expected because of (1) differing thermal conditions across the part geometries, (2) the LM used for melting, (3) the spatial location of the samples across the build area, (4) the spatial location of the samples in the vertical build direction, (5) the effects of overhangs on the thermal history of the samples, and (6) the effects of overhangs on the surface roughness of a subset of the samples. Figure 14 shows a representative in situ image from one layer of the build, and Figure 15 shows the completed build before the heat-treatment operation.

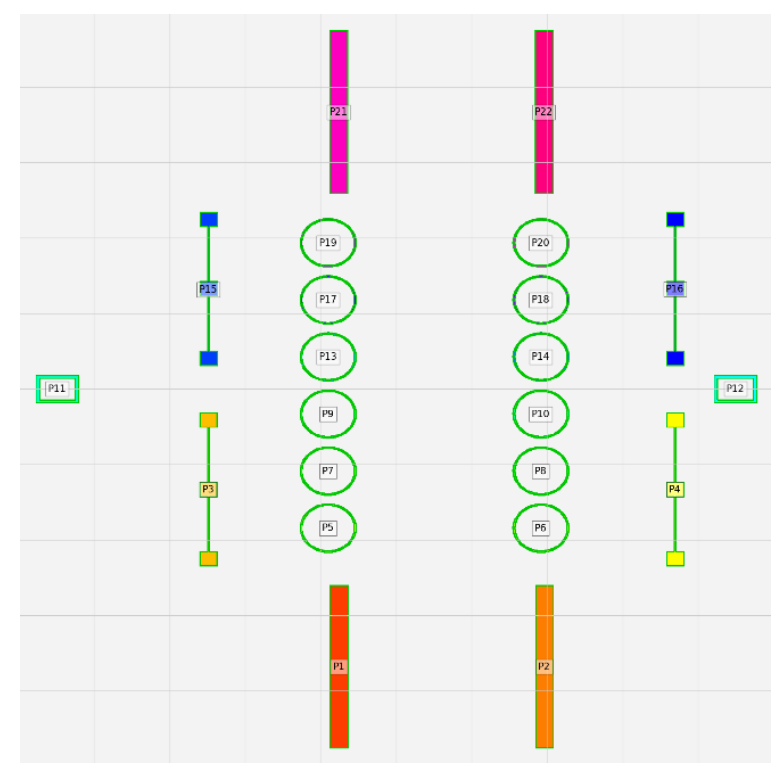

Figure 12. The build layout for "TCR Phase 1 Build 3" as viewed from the top with the Peregrine part identifiers overlaid.

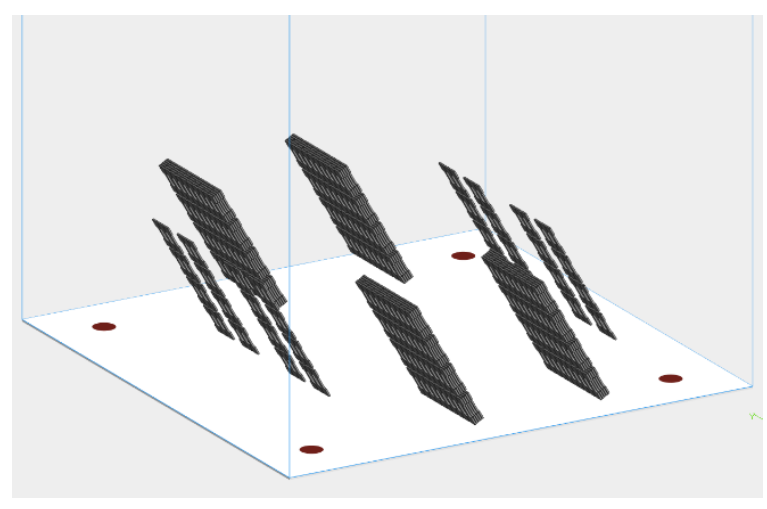

Figure 13. CAD rendering of all the SS-J3 samples that could be extracted from "TCR Phase 1 Build 3." 


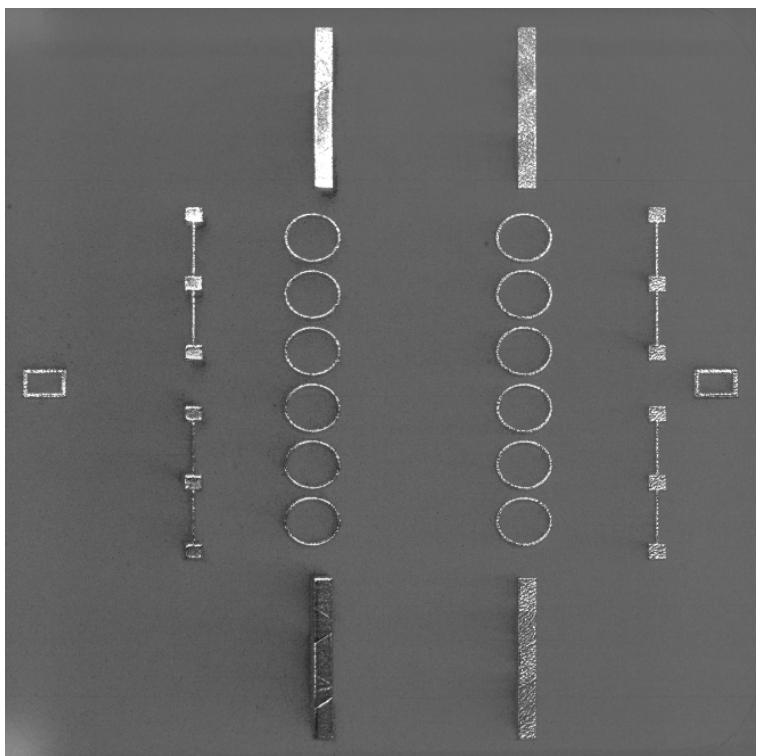

Figure 14. One in situ visible-light camera image of the powder bed captured during "TCR Phase 1 Build 3."

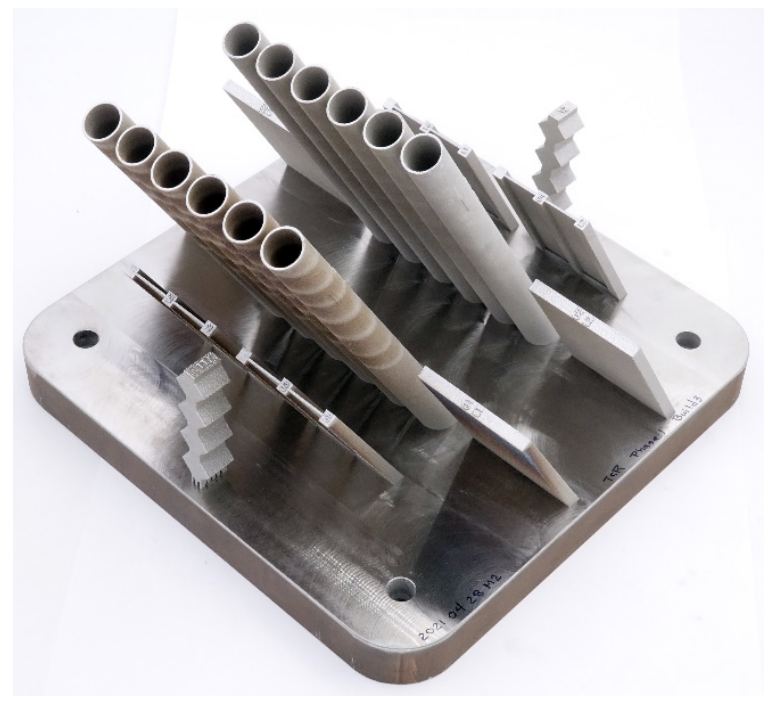

Figure 15. Isometric view of "TCR Phase 1 Build 3" immediately after the printing operation.

Build 2021-08-03, “TCR Phase 1 Build 4," was designed to capture data on components exposed to excessive soot deposition. This build contained 77 parts (Figure 16) from which a maximum of 992 SS-J3 samples could be extracted (Figure 17). Sixteen burst tubes, two powder sampling containers, four XCT specimens, and one thermocouple well were also printed. The SS-J3 samples were sourced from four clusters of as-printed parts, and each cluster comprised one each of the SSJ3-A, SSJ3-B, and SSJ3-C part geometries. The burst tubes and XCT specimens were evenly distributed among the clusters. All SS-J3 samples and burst tubes were printed using the NOMINAL process parameters with two clusters printed with LM-1 and two clusters printed with LM-2. All clusters were printed with the contour process parameters provided by Concept Laser with an offset-to-contour value of $110 \mu \mathrm{m}$. All the parts enumerated previously - except for the thermocouple well-were placed immediately downstream from "soot dumper" parts. These parts were printed with the SOOT process parameter set, which was developed specifically to generate large quantities of soot. At a build height of $42.55 \mathrm{~mm}$, the shield gas flow rate was intentionally reduced by manually changing the target ventilator gas flow rate from its standard setpoint of $40 \mathrm{~m}^{3} / \mathrm{h}$ to $25 \mathrm{~m}^{3} / \mathrm{h}$. This change was designed to further increase the amount of soot deposited on the parts and simulate the effects of a gas filter nearing the end of its life. Variations in the in situ sensor signatures, process anomalies, and tensile properties are expected because of (1) differing thermal conditions across the part geometries, (2) the LM used for melting, (3) the spatial location of the samples across the build area, (4) the spatial location of the samples in the vertical build direction, and (5) the increased levels of soot deposition and the resulting inclusions and microstructural changes due to the adjacent soot dumper parts and the reduction in the shield gas flow rate. Figure 18 shows a representative in situ image from one layer of the build, and Figure 19 shows the completed build before the heat-treatment operation. 


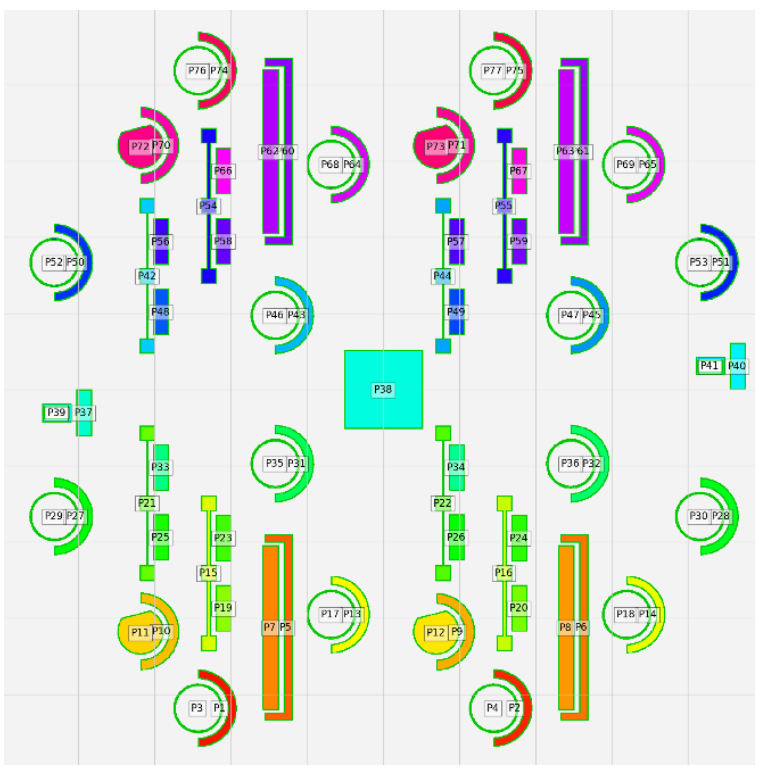

Figure 16. The build layout for "TCR Phase 1 Build 4" as viewed from the top with the Peregrine part identifiers overlaid.

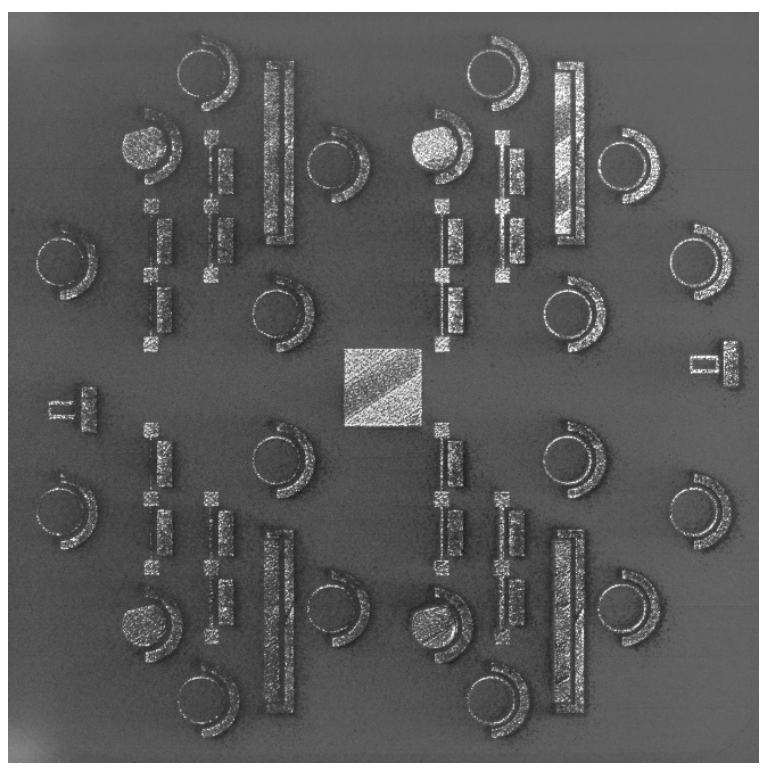

Figure 18. One in situ visible-light camera image of the powder bed captured during "TCR Phase 1 Build 4."

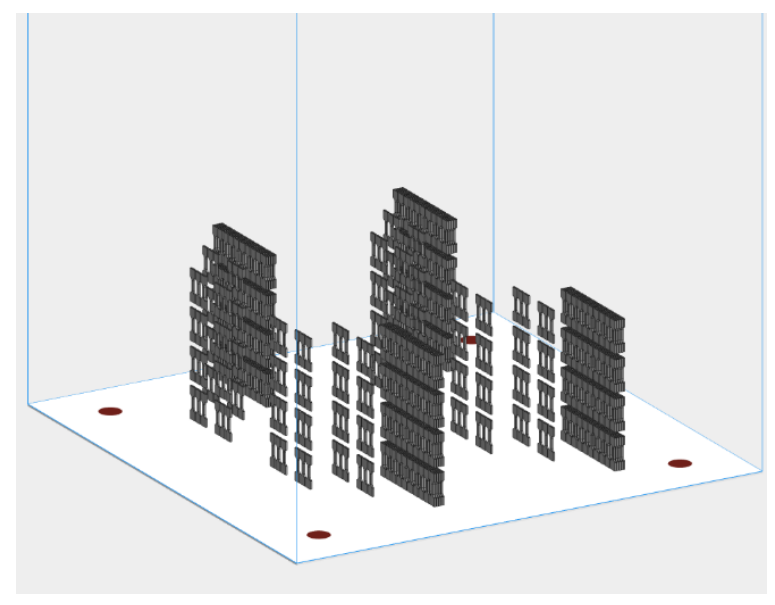

Figure 17. CAD rendering of all the SS-J3 samples that could be extracted from "TCR Phase 1 Build 4."

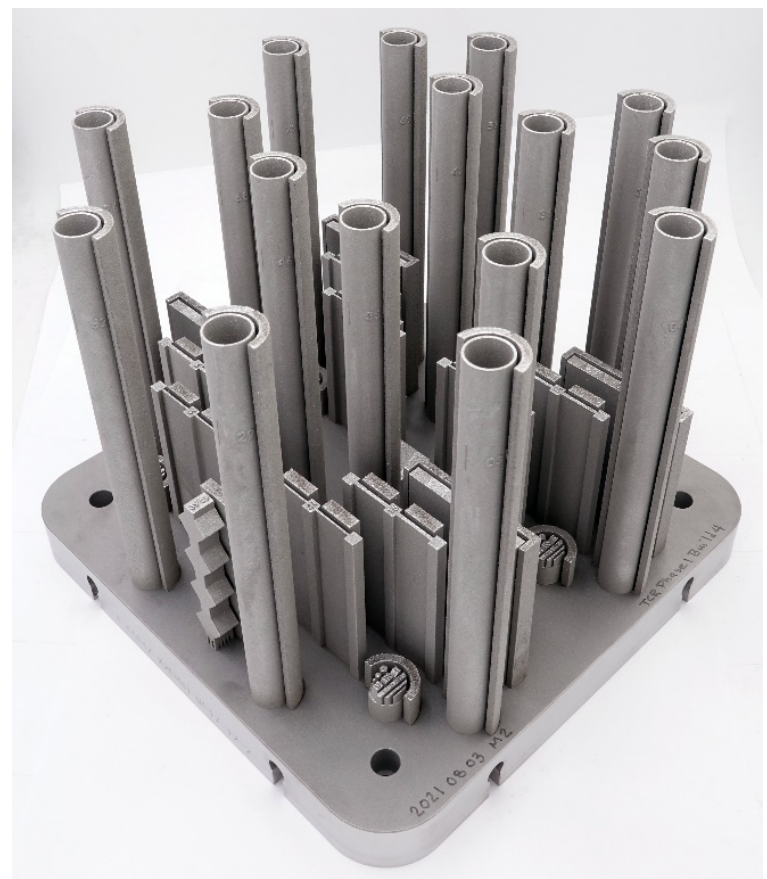

Figure 19. Isometric view of "TCR Phase 1 Build 4" immediately after the printing operation.

Build 2021-08-23, “TCR Phase 1 Build 5," was designed to capture data on components that experience recoater streaking and incomplete spreading events. This build contained 39 parts (Figure 20) from which a maximum of 3,296 SS-J3 samples could be extracted (Figure 21). Sixteen burst tubes, two powder 
sampling containers, four XCT specimens, and one thermocouple well were also printed. The SS-J3 samples were sourced from four clusters of as-printed parts, and each cluster comprised one each of the SSJ3-A, SSJ3-B, SSJ3-C, and SSJ3-D part geometries. The burst tubes and XCT specimens were evenly distributed among the clusters. All SS-J3 samples and burst tubes were printed with contours and using the NOMINAL process parameters with an offset-to-contour value of $110 \mu \mathrm{m}$. Two clusters were printed with LM-1, and two clusters were printed with LM-2. This build was printed with an intentionally damaged (nicked) recoater blade to induce significant recoater streaking artifacts throughout the SS-J3 part geometries and burst tubes. At build heights of $33.05 \mathrm{~mm}$ and $52.00 \mathrm{~mm}$, which correspond approximately to the centers of two sets of SS-J3 sample gauge sections, the powder dosing factor was manually lowered from $200 \%$ to $5 \%$ to intentionally induce incomplete spreading and the resulting superelevation anomalies for between one and three layers, depending on the cluster. Variations in the in situ sensor signatures, process anomalies, and tensile properties are expected because of (1) differing thermal conditions across the part geometries, (2) the LM used for melting, (3) the spatial location of the samples across the build area, (4) the spatial location of the samples in the vertical build direction, (5) recoater streaking anomalies intersecting with a subset of the parts, and (6) incomplete spreading inducing superelevation anomalies within a subset of the parts. Figure 22 shows a representative in situ image from one layer of the build, and Figure 23 shows the completed build before the heat-treatment operation.

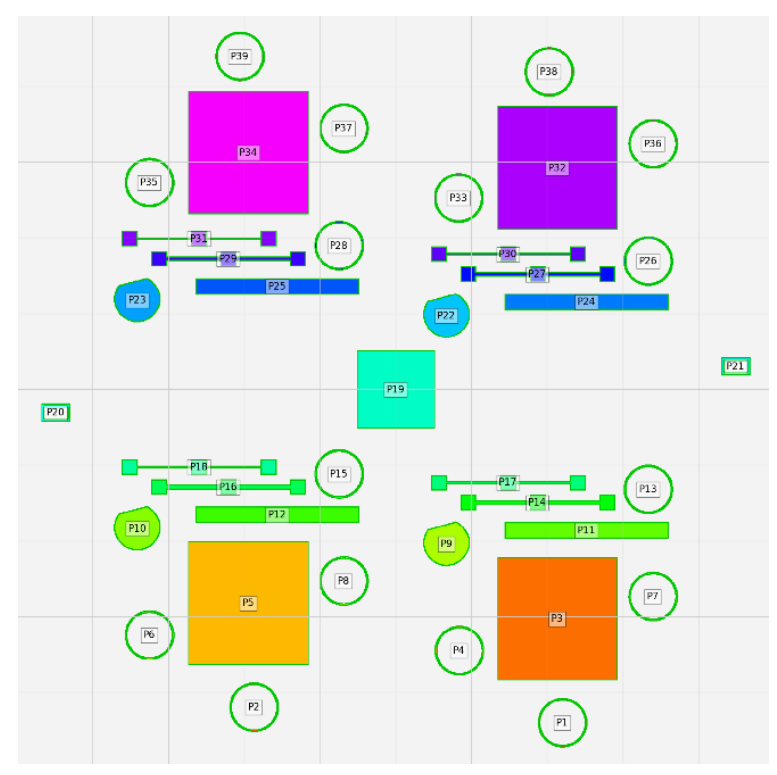

Figure 20. The build layout for "TCR Phase 1 Build 5" as viewed from the top with the Peregrine part identifiers overlaid.

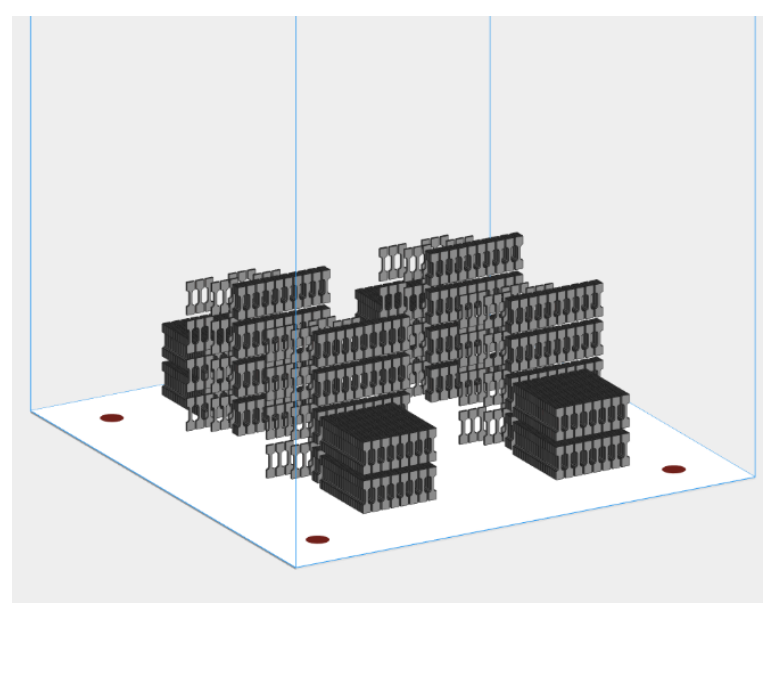

Figure 21. CAD rendering of all the SS-J3 samples that could be extracted from "TCR Phase 1 Build 5." 


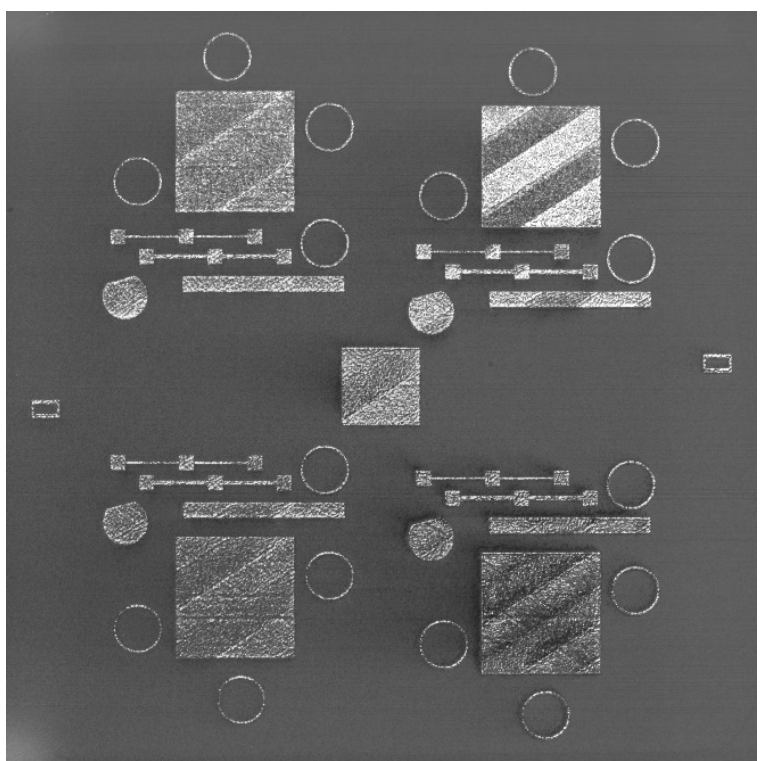

Figure 22. One in situ visible-light camera image of the powder bed captured during "TCR Phase 1 Build 5."

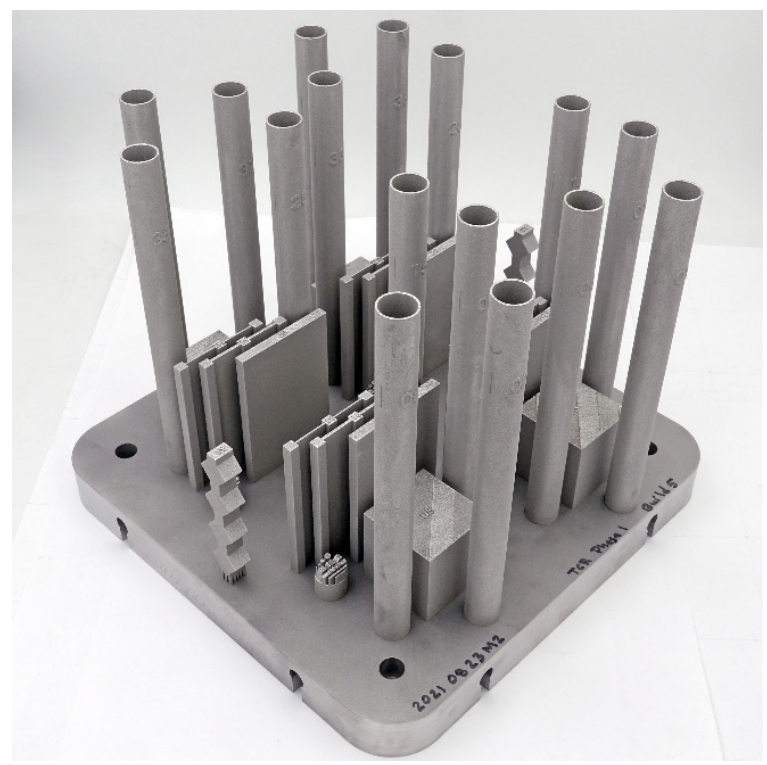

Figure 23. Isometric view of "TCR Phase 1 Build 5" immediately after the printing operation.

\subsection{HEAT TREATMENT}

All test campaign builds were heat-treated to relieve the residual stresses that are induced during L-PBF manufacturing. The greater the residual stresses, the more distortion the printed parts are expected to undergo during sample extraction. Such distortion reduces the registration fidelity between the ground truth tensile data and the in situ sensor data and can strongly impact the dimensional tolerances of the SSJ3 samples. Stress-relieving heat treatments are an industry standard postprocessing step for L-PBF manufactured components and would be performed on any SS 316L nuclear core components.

The degree to which the residual stresses can be reduced post-build is a strong function of the heattreatment soak temperature and a weaker function of the heat-treatment soak time. Therefore, the nominal soak temperature was set to the maximum in-specification value of $650^{\circ} \mathrm{C}$, as defined by Li Wei-Ying Chen et al. [9] in which L-PBF SS 316L creep properties were evaluated over a range of heat-treatment temperature profiles. Several soak times ranging from $30 \mathrm{~min}$ to $24 \mathrm{~h}$ were then tested, and a nominal soak time of $24 \mathrm{~h}$ was selected because it resulted in the least part distortion without unreasonably lengthening the postprocessing timeline. All heat treatments were performed on the IR Flatbed X189828 furnace at ORNL's main campus (building 4508) in an ambient air environment and using the furnace profile described in Table 6 . To ensure that the bulk parts reach the target temperatures, an additional monitoring thermocouple is always inserted into a well inside a $1 \mathrm{in}$. cube printed in the center of each build plate. After heat treatment, the build plate and parts are bead-blasted to remove scaling and ensure accurate touch-off points for the wire EDM.

Table 6. Furnace profile used for all TCR FY21 test campaign builds.

\begin{tabular}{cc}
$\begin{array}{c}\text { Segment } \\
\text { number }\end{array}$ & Segment type \\
\hline 1 & Ramp up at $10^{\circ} \mathrm{C} / \mathrm{min}$ to $650 \pm 10^{\circ} \mathrm{C}$ \\
2 & Soak at $650 \pm 10^{\circ} \mathrm{C}$ for $24 \pm 0.5 \mathrm{~h}$ \\
3 & Furnace-cool to $100 \pm 20^{\circ} \mathrm{C}$
\end{tabular}




\subsection{SAMPLE EXTRACTION}

The SS-J3 tensile samples are printed within as-built part geometries designated as SSJ3-A, SSJ3-B, SSJ3-C, and SSJ3-D. To extract the samples from the parts, the parts are first removed from the build plate using the Sodick AQ750LH wire EDM at the MDF. Each individual part is then fixtured, and "bread slices" (Figure 24) are cut along the vertical build direction to separate the SS-J3 samples into sheets. The sheets are then re-fixtured, and the wire EDM is used to cut the profiles of the SS-J3 samples; a small tab is left uncut so that the samples remain attached to the sheet for tracking purposes. The wire EDM cut paths are programmed by using a standard computer aided machining (CAM) package. The nominal locations of the SS-J3 samples are predefined in a CAD model, and the coordinate origins of the parts are carefully selected to enable registration of the SS-J3 samples with the in situ data. Finally, each sheet is labeled and handed off for tensile testing at which point the tabs will be snipped immediately before testing, and individual labels for each sample will be applied.

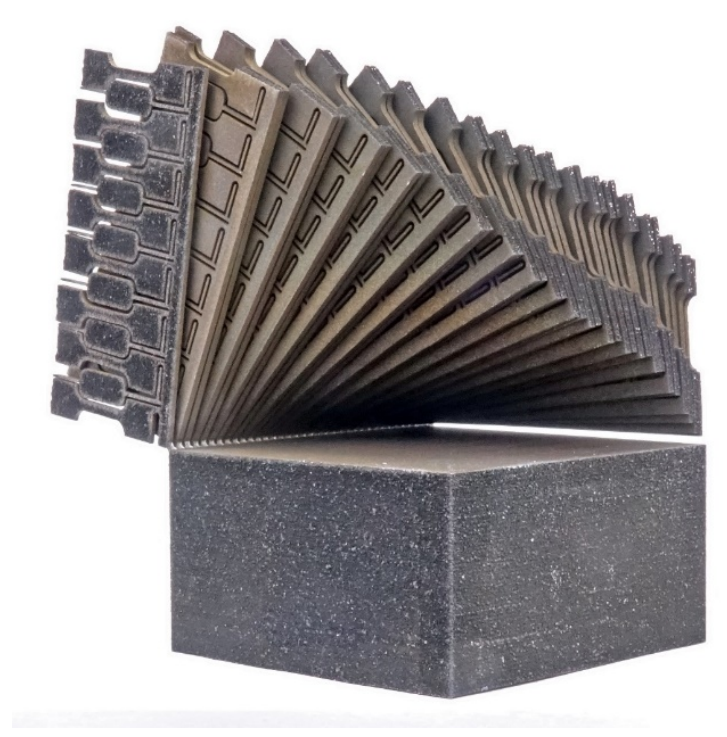

Figure 24. A set of L-2 SS-J3 samples during extraction from an SSJ3-D geometry, still contained within their corresponding bread slices.

\subsection{SAMPLE TRACKING}

The digital thread of each tensile sample begins when the L-PBF operation (Figure 25) is instantiated in the database via the MDF Digital Tool (DT) web client. After the collected metadata are validated, a storage structure is created for the dataset to facilitate the archival process. The database is then updated, and a globally unique identifier is assigned to the build dataset. Each as-built part and each possible child sample are automatically assigned a build-wise unique identifier by Peregrine as described in Scime et al. [6], and this information is shared with the database via a dedicated application programming interface (API). Next, the heat-treatment operation (Section 2.3) is performed on the entire build, and the associated metadata are recorded using the DT; both the target and measured thermal profiles are also uploaded to the DP via the DT. After heat treatment, the as-built parts are removed from the build plate using a wire EDM, as described in Section 2.4. During this stage, the parts are instantiated within the database and assigned globally unique identifiers. Similarly, the samples are only instantiated in the 
database and assigned globally unique identifiers after their corresponding wire EDM extraction operation is recorded by the DT.

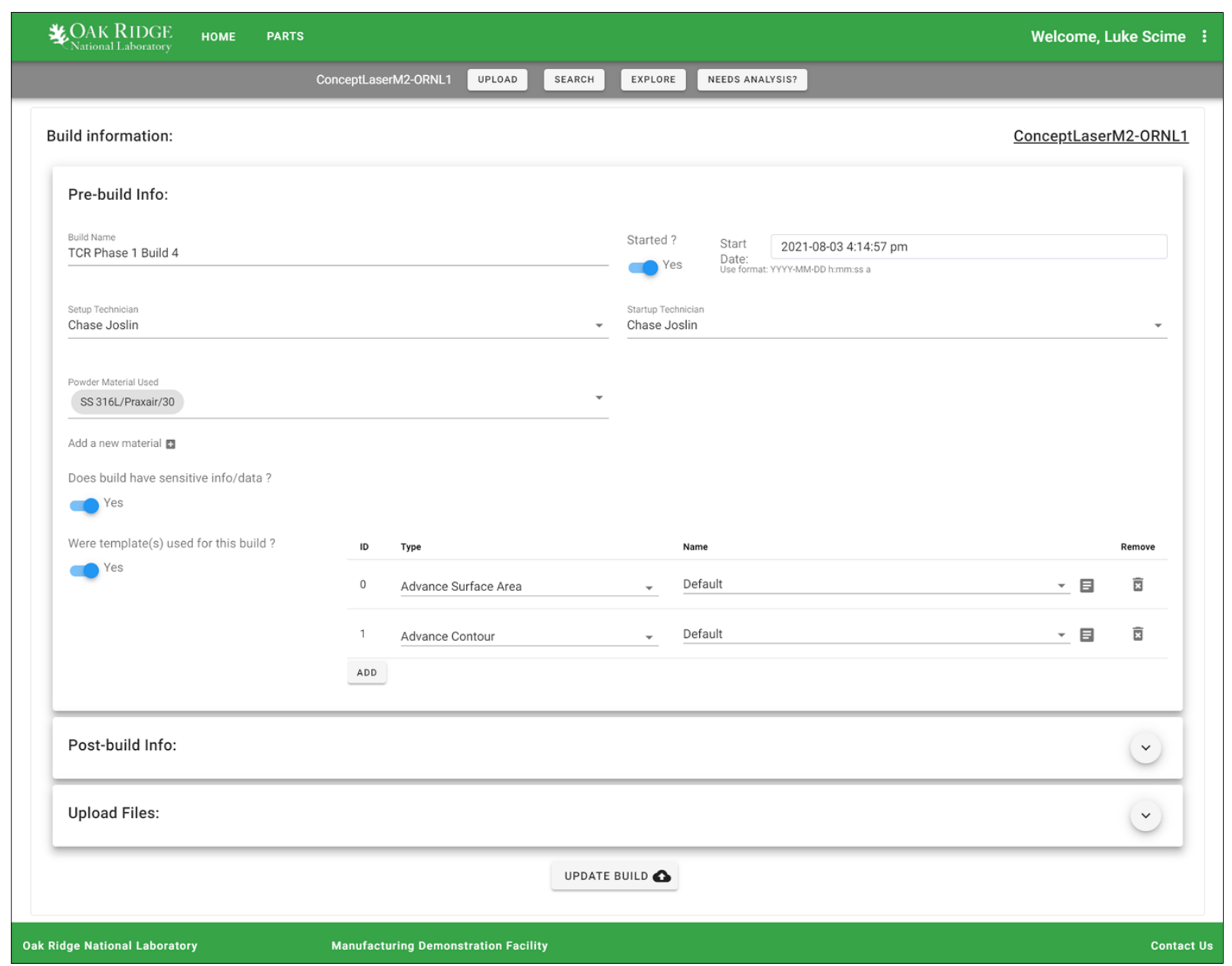

Figure 25. The DT web portal for instantiating a Concept Laser M2 L-PBF operation. Metadata are entered by the printer technician and validated against accepted data ranges and types.

As each sample is removed from the bulk printed material, it is placed in an individual plastic bag labeled with a quick response $(\mathrm{QR})$ code that contains the metadata required to uniquely identify the sample throughout the tensile testing operation described in Section 3.1. Finally, in addition to collecting metadata for the digital threads, the DT and its associated APIs can also be used as a catalogue for searching through historical datasets to support quality assurance efforts, as shown in Figure 26. 


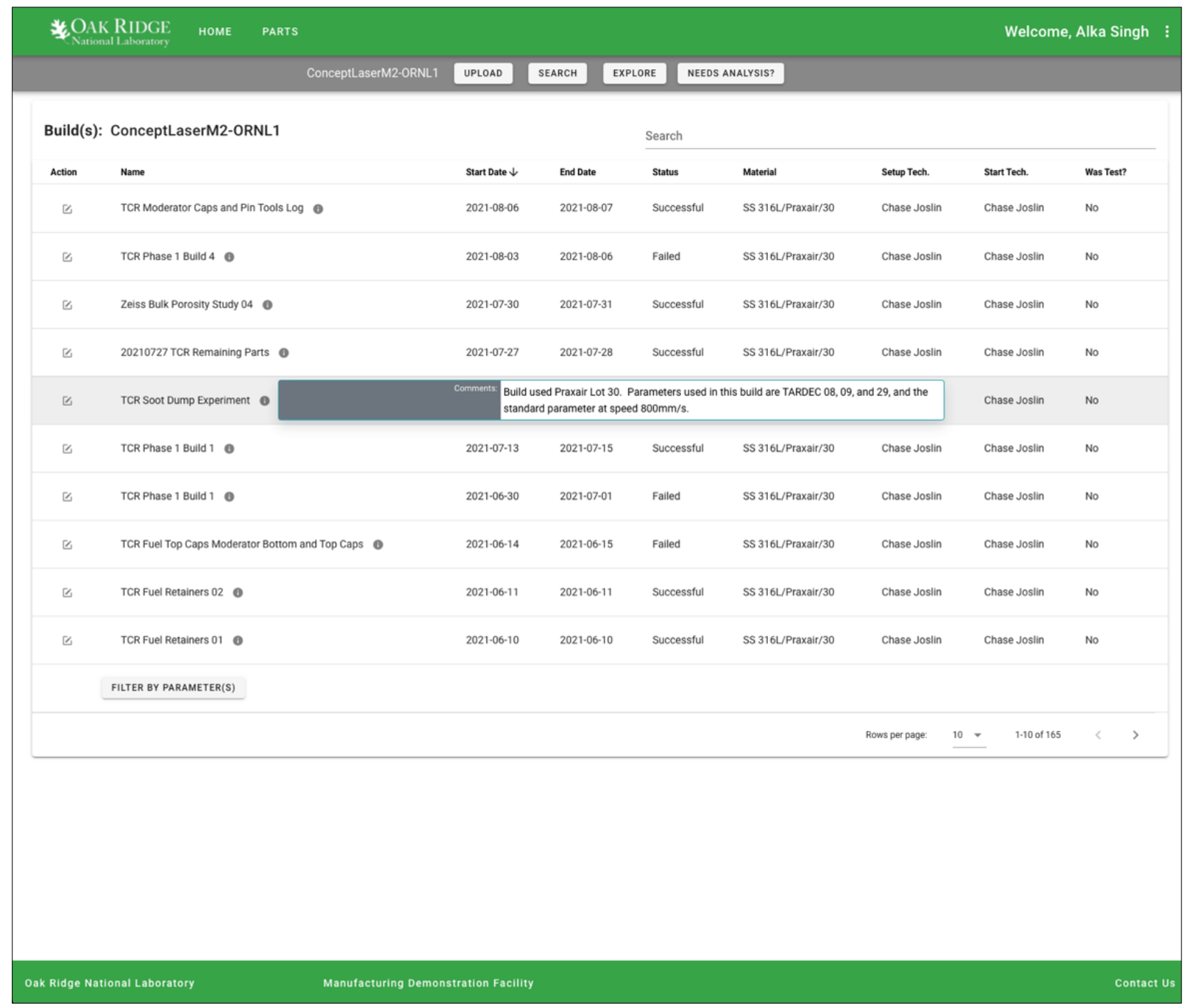

Figure 26. An example of DT search results for the Concept Laser M2 L-PBF operations.

\section{CHARACTERIZATION AND TESTING}

\subsection{TENSILE TESTING SETUP AND PROCEDURES}

Tensile testing was conducted in accordance with the ASTM E8/E8M standard [10]. The SS-J3 samples were tested using one of two TestResources single-controller dual-frame servo-electric systems (frame models 800LE3 and 810LE5) equipped with $500 \mathrm{lbf}$ static-rated load cells calibrated to the ASTM E4 standard [11]. The gauge section width and thickness of each SS-J3 sample were measured using calipers. Although the gauge sections were consistently well machined, slight tapers were present, so the thinnest section of the gauge was reported because this was the region most likely to fail during testing. After measuring, the samples were installed in the test system utilizing a set of shoulder-loading tensile grips. The samples were then preloaded, generally between $10 \mathrm{~N}$ and $50 \mathrm{~N}$, with occasional overshoots; no reported samples were preloaded beyond their yield load. The appropriate metadata were written to the test file, then the samples were loaded in displacement control at $0.5 \mathrm{~mm} / \mathrm{min}(\sim 10 \%$ strain per minute, as measured by crosshead displacement) at a data acquisition rate of $10 \mathrm{~Hz}$. The load and crosshead displacement were recorded as a time series. The samples were loaded until either final fracture or the 
load dropped below $10 \mathrm{~N}$. The failed SS-J3 samples were then returned to their individual plastic bags and preserved for future analyses. Figure 27 shows an example engineering stress-strain curve and highlights the tensile properties of interest, which include the ultimate tensile strength (UTS), yield tensile strength (YTS), total elongation (TE), and uniform elongation (UE).

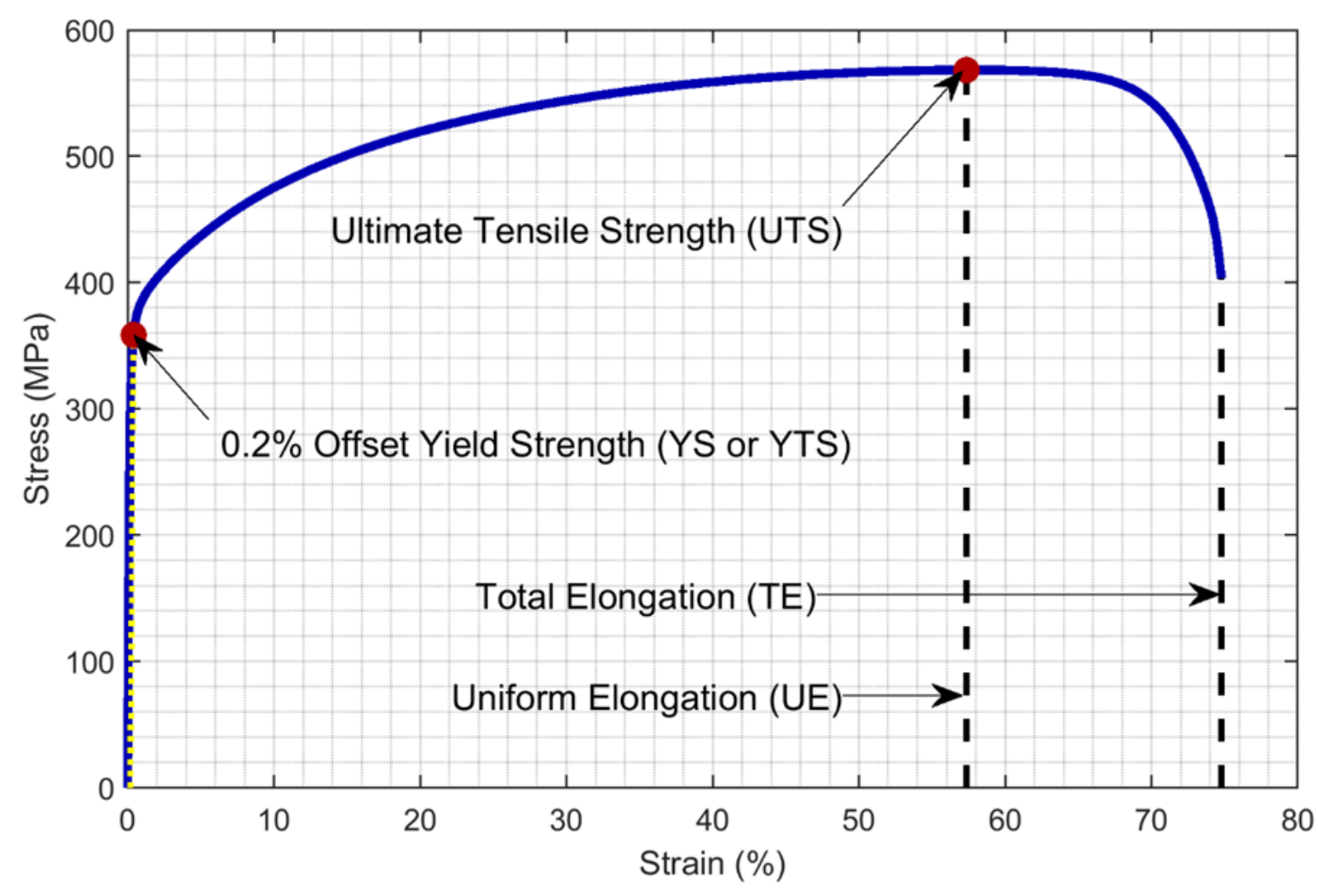

Figure 27. Example AM SS 316L stress-strain curve with annotations highlighting the tensile properties of interest.

To obtain the tensile properties marked in Figure 27, the load-displacement data are first smoothed to eliminate the noise and fluctuations encountered during the testing process, and extraneous points are removed. Stress values are obtained by using Eq. (1):

$$
\sigma_{i}=\frac{F_{i}}{A}
$$

where $\sigma_{i}$ is the stress at data point, $i ; F_{i}$ is the force or load at $i$; and $A$ is the cross section of the gauge section of the tensile sample. Although stress values can be calculated directly, measuring the strain is more involved. First, the load line compliance, $C_{L L}$, must be calculated for each tensile test. The load line compliance can vary significantly between not only different load frames but also different tests performed on the same system. The load line compliance is calculated as the inverse of the slope of the elastic region of the load-displacement curve. The elastic component of each displacement point is then calculated as shown in Eq. (2):

$$
\delta_{e(i)}=C_{L L} F_{i}
$$


This value is then subtracted from each displacement value, $\delta_{i}$, so that only the plastic component, $\delta_{p(i)}$, of each displacement value remains (points corresponding to the linear elastic region may be truncated). To obtain only the elastic component of the gauge section displacement, the compliance of the SS-J3 gauge section, $C_{G}$, must be calculated as shown in Eq. (3):

$$
C_{G}=\frac{L}{A E}
$$

where $L$ is the length of the gauge section, and $E$ is the elastic modulus taken from literature as $193 \mathrm{GPa}$ [12]. The elastic displacement of the gauge section is then calculated as shown in Eq. (4):

$$
\delta_{e, G(i)}=C_{G} F_{i} .
$$

The gauge elastic displacement at each point, $i$, is added to the plastic displacement, $\delta_{p(i)}$, to calculate the total gauge section displacement, $\delta_{G(i)}$. Strain, $\varepsilon_{i}$, is then calculated as shown in Eq. (5):

$$
\varepsilon_{i}=\frac{\delta_{G(i)}}{L} .
$$

With the $\sigma_{i}$ and $\varepsilon_{i}$ values calculated and an additional data point inserted at the origin, the stress-strain curve can be constructed, and the pertinent strength and elongation values can be extracted. The calculation of the tensile values of interest was automated using a script written in Python. The script takes as input a comma separated value (CSV) file, which contains the displacement and load time series and the measured gauge area of the individual sample. The elastic region is considered to be the region of the load-displacement curve with the greatest slope. Barring any errors or anomalies in the data, this generally occurs immediately before the elastic-plastic transition. Although higher slopes might occur at lower displacements than this, these are often caused by mistakes in the test procedure, such as preload overloading, and it is critical that the true elastic region be distinguished from these regions. Once the elastic region is determined, the load-displacement curve is converted to a stress-strain curve by using Eqs. (1)-(5), and the tensile properties of interest are calculated. The YTS values are calculated as the $y$ intercept of the stress-strain curve and the $0.2 \%$ strain offset line (i.e., a line with slope $E$ and an $x$ intercept of $0.2 \%$ strain). The UTS and UE values are the respective stress and strain values at the maximum of the stress-strain curve, and TE is the strain at breakage.

\subsection{TENSILE TESTING RESULTS AND OBSERVATIONS}

In FY21, approximately 3,000 samples were tested as part of the AIR test campaign. Only samples from the "TCR Phase 1 Build 2" and "TCR Phase 1 Build 3" builds were tested at the time of this report. This section highlights some of the tensile test results and discusses some of the key inter-build, inter-part, and intra-part differences observed in the tensile properties.

Figure 28 shows selected stress-strain curves from samples extracted from the SSJ3-C geometries printed in the "TCR Phase 1 Build 2" build and designated P11, P12, P24, and P25, each respectively built with the NOMINAL, KEYHOLE, BEST, and LOF process parameter sets. The curves are from samples S26, S76, S126, and S176 from each part, which are all located at the planar center of their respective parts. This allows for direct comparison between samples printed with different process parameters and in different vertical locations within the build volume. 


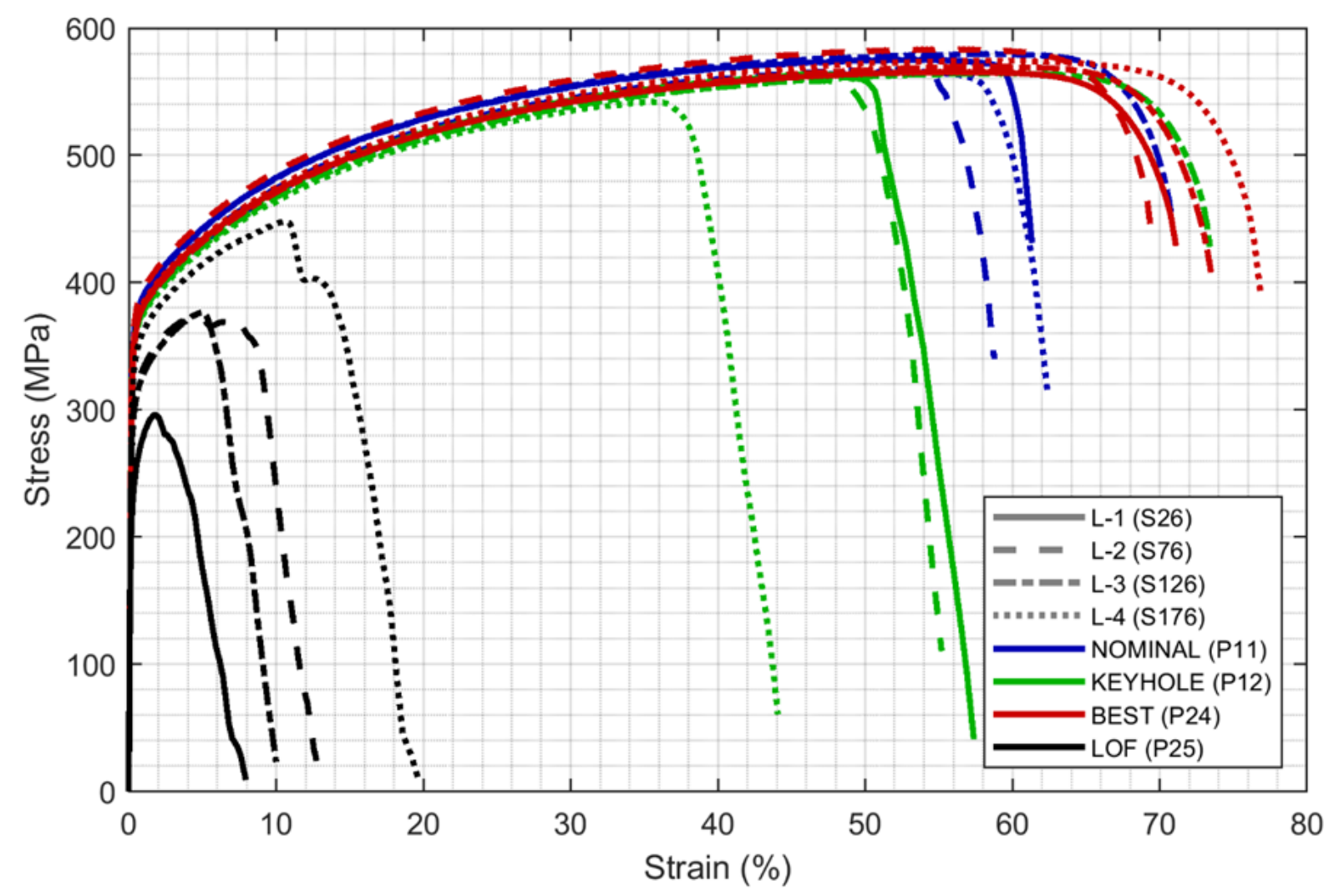

Figure 28. Selected stress-strain curves from samples extracted from the SSJ3-C geometries printed in the "TCR Phase 1 Build 2" build. All samples were located near the planar centerline of their respective parts and at the four different build heights designated L-1, L-2, L-3, and L-4.

The mechanical behavior is as expected for this build, with the best to worst processing parameters found to be BEST, NOMINAL, KEYHOLE, and LOF. P24 (OPTIMAL) shows the most consistent behavior and the highest ductility. Interestingly, the tensile behaviors for P11.S126 (NOMINAL) and P12.S126 (KEYHOLE) are similar to those of the samples extracted from P24 (OPTIMAL). This behavior might be driven by the SSJ3-C parts' proximity to the SSJ3-D parts at the L-1 and L-2 build heights. At L-3 and L4 , the SSJ3-D part is no longer being printed, possibly influencing the thermal history of the samples embedded in the SSJ3-C parts and affecting their mechanical properties.

Based on the results in Figure 28, the measured mechanical properties of these materials appear to primarily depend on the prevalence of flaws generated during the build process. Before the UE of each stress-strain curve is reached, each curve follows approximately the same stress-strain path, indicating there is no fundamental difference in the work-hardening behavior between P11, P12, and P24; P25 samples show little to no work-hardening because they fail at extremely low strains. However, beyond their respective UEs, P11 and P12 show much steeper declines in stress than P24; this also consistently occurs at a lower strain with P12 than with P11. This suggests flaw densities within P11, P12, and P25, which are sufficient to reduce their measured mechanical properties relative to P24.

The region of greatest flaw density in each sample heavily influences the location within the gauge section where the sample will fail. High flaw density regions effectively reduce the cross section of the SS-J3 gauge. This is evidenced by not only lower failure strains but also by lower fracture energies, as P11, P12, and P25 generally show a greater stress reduction before final fracture, indicating that the strain energy is being dissipated before final fracture. Because the high flaw density regions of each sample 
have a reduced effective cross section, they will locally reach maximum work-hardening before regions of lower flaw density. Because the lower flaw density regions can still be work-hardened, they can dissipate mechanical energy from additional straining, leading to a lower final fracture energy.

In contrast, samples from P24 and samples P11.S126 and P12.S126 do not show as steep of a decline in stress beyond UE, and their final fracture energies are much higher. This indicates that the flaw densities in these samples are low enough that the mechanical properties are uniform throughout the gauge section. This also suggests that the gauge section work-hardens uniformly so that beyond the UE, the capacity of the non-necked regions to dissipate energy through plastic deformation is minimal. In these instances, mechanically induced microvoid (on the order of $0.5 \mu \mathrm{m}-5 \mu \mathrm{m}$ ) coalescence is the final fracture mechanism [13], which results in more consistent failure behavior than failure resulting from preexisting production-generated flaws.

Figure 29 shows selected stress-strain curves for samples taken from SSJ3-C as-built parts printed using the BEST process parameter set in builds "TCR Phase 1 Build 2" and "TCR Phase 1 Build 3." Based on the results in Figure 28, parts produced using the BEST parameter sets are used for this comparison because their measured properties are the most consistent and do not appear to be influenced by production-generated defects. In this comparison, the variable of interest between the "TCR Phase 1 Build 2" and "TCR Phase 1 Build 3" builds is part orientation with the parts in "TCR Phase 1 Build 2" built parallel to the vertical build direction and parts in "TCR Phase 1 Build 3" printed at $30^{\circ}$ relative to the vertical build direction (Section 2.2). Based on the results in Figure 29, the measured intra-part tensile properties are relatively consistent, and P1 and P21 from "TCR Phase 1 Build 3" also show inter-part consistency. Regarding inter-build differences, "TCR Phase 1 Build 2" shows somewhat higher ductility and lower strength than "TCR Phase 1 Build 3," although every sample presented in Figure 29 shows excellent tensile properties and high-energy final fracture, indicating a low prevalence of productionrelated defects. 


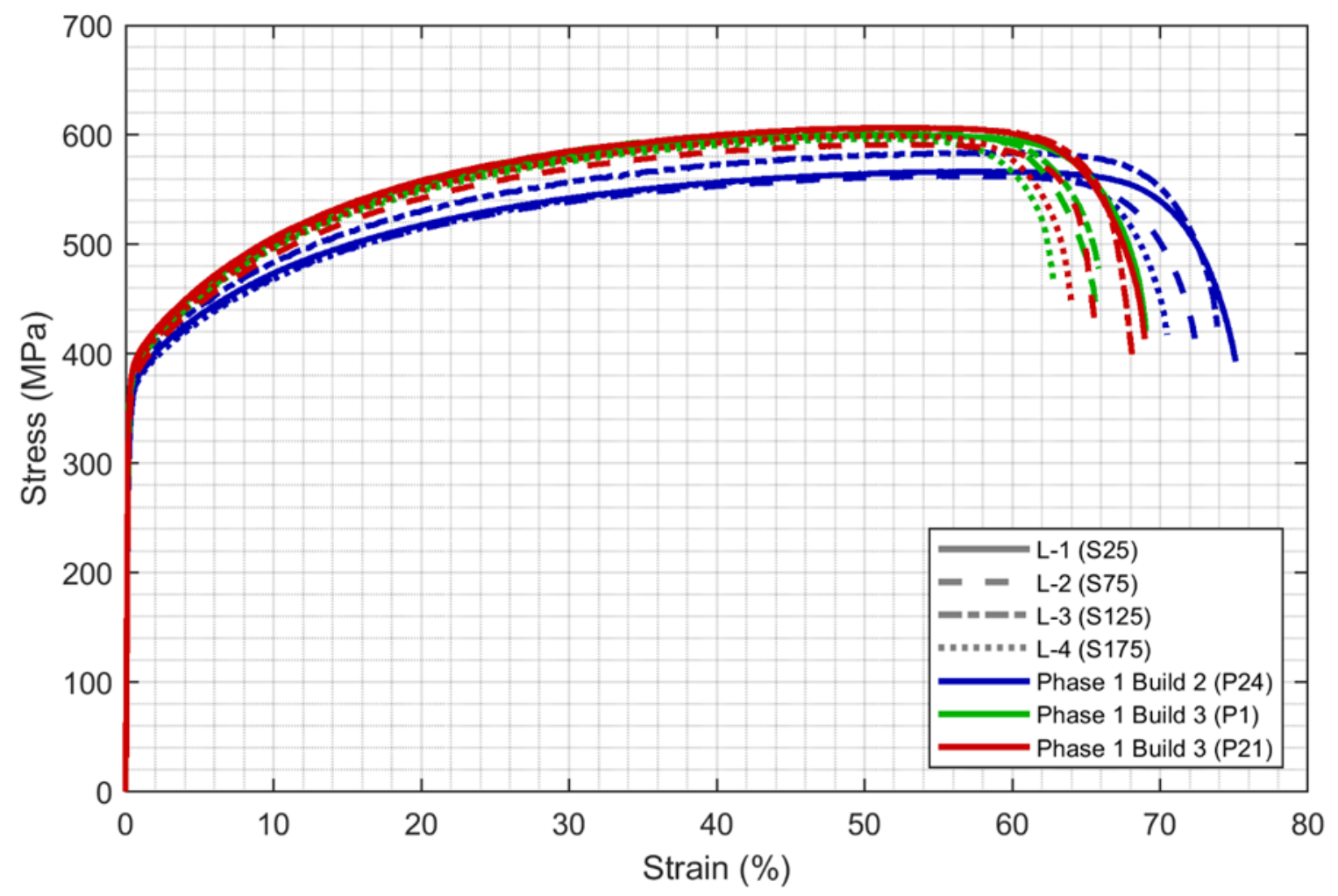

Figure 29. Selected stress-strain curves for samples taken from SSJ3-C as-built parts printed using the BEST process parameter set in builds "TCR Phase 1 Build 2" and "TCR Phase 1 Build 3." Parts in "TCR Phase 1 Build 2" were built parallel to the vertical build direction, and parts in "TCR Phase 1 Build 3" were printed at $30^{\circ}$ relative to the vertical build direction.

It is well understood that the orientation of a sample can significantly influence its tensile properties, particularly its orientation relative to the vertical build direction [14-20]. Because of the large variability in processing parameters, the relationship between orientation and tensile properties can vary considerably. For example, consistent with the results of this study, the results reported in Im et al. [13] and Charmi et al. [14] show an increase in strength and a decrease in ductility with increasing angle relative to the build direction. However, the consistency of the results of this study to those in other works $[14,16,19,20]$ is tenuous, and there is significant variation among the conclusions presented in those same prior works, suggesting that this remains an open research question. To properly explain the behavior observed in Figure 29, a microstructural analysis is required to determine the most influential microstructural features and determine whether there are any fundamental differences in the microstructures between the "TCR Phase 1 Build 2" and TCR Phase 1 Build 3" parts.

Figure 30 shows selected stress-strain curves of samples from "TCR Phase 1 Build 2" P24, which is an SSJ3-C geometry. Curves from samples from the interior of the part and the two large exterior faces are featured. The part is oriented so that its largest faces are parallel to the $x-z$ plane of the build with the front and back faces nominally separated by $5 \mathrm{~mm}$ in the $y$-direction. The behavior observed in Figure 30 is expected, and the interior samples show higher strength and ductility relative to the surface samples due to high near-surface porosity. The samples from the back face also show improved tensile properties over those from the front face. In this case, the samples from the front face were machined much thinner than nominal $(0.62 \mathrm{~mm}-0.73 \mathrm{~mm})$, whereas those from the back face were machined much thicker $(0.97 \mathrm{~mm}$ $-1.05 \mathrm{~mm}$ ) and thus contained more of the dense interior, which might have contributed to the improvement in measured tensile properties relative to the front face samples. Although it is apparent that 
near-surface porosity affects the measured tensile properties relative to the dense interior, the significant differences in sample machining are a convoluting factor that prevents the authors from drawing a conclusion regarding any fundamental differences between the properties of the front and back surfaces.

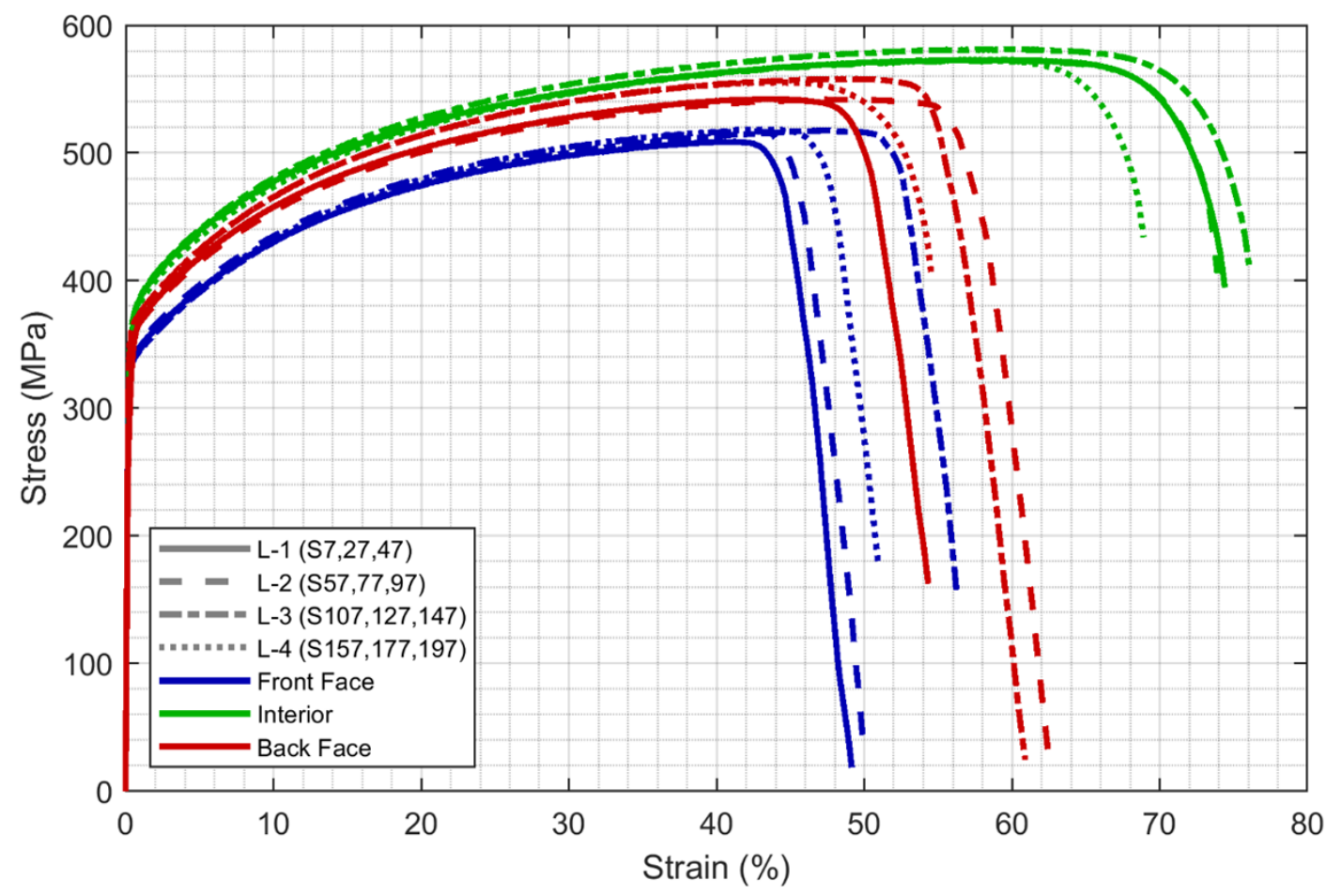

Figure 30. Selected stress-strain curves from "TCR Phase 1 Build 2" P24, featuring both interior tensile sample data and near-surface tensile sample data.

Figure 31 shows selected stress-strain curves of samples from SSJ3-C (P24) and SSJ3-B (P27) parts printed using the BEST process parameter set during the "TCR Phase 1 Build 2" build. This comparison is designed to highlight how differences in as-built part thickness and geometry affect the tensile properties. Although an additional SSJ3-A part (P30) was printed with the BEST parameter set in "TCR Phase 1 Build 2," the as-printed faces were not machined away from the samples, and it can be inferred from Figure 39 that the measured properties of P30 would be relatively low and dominated by nearsurface porosity. In contrast, P27 shows excellent tensile properties, exceeding P24 in both strength and ductility. The slopes of the stress-strain curves of P27 are also higher than those of P24, indicating that the work-hardening rates of the two parts are different and thus there is a fundamental difference in the microstructure between the two parts exists. These microstructural differences are likely the result of differences in the cooling rates between the two parts. Because P27 is much thinner than P24, there is less thermal mass to absorb and conduct heat away from the hot build surface. Therefore, P27 is expected to cool more slowly than P24, leading to differences in microstructure and therefore mechanical properties. The dislocation network, grain size, $\delta$-ferrite content, and ceramic precipitate content are some features that could be affected by differences in the cooling rate. However, determining the dominant features or mechanisms involved would require an in-depth microstructural investigation of these parts. 


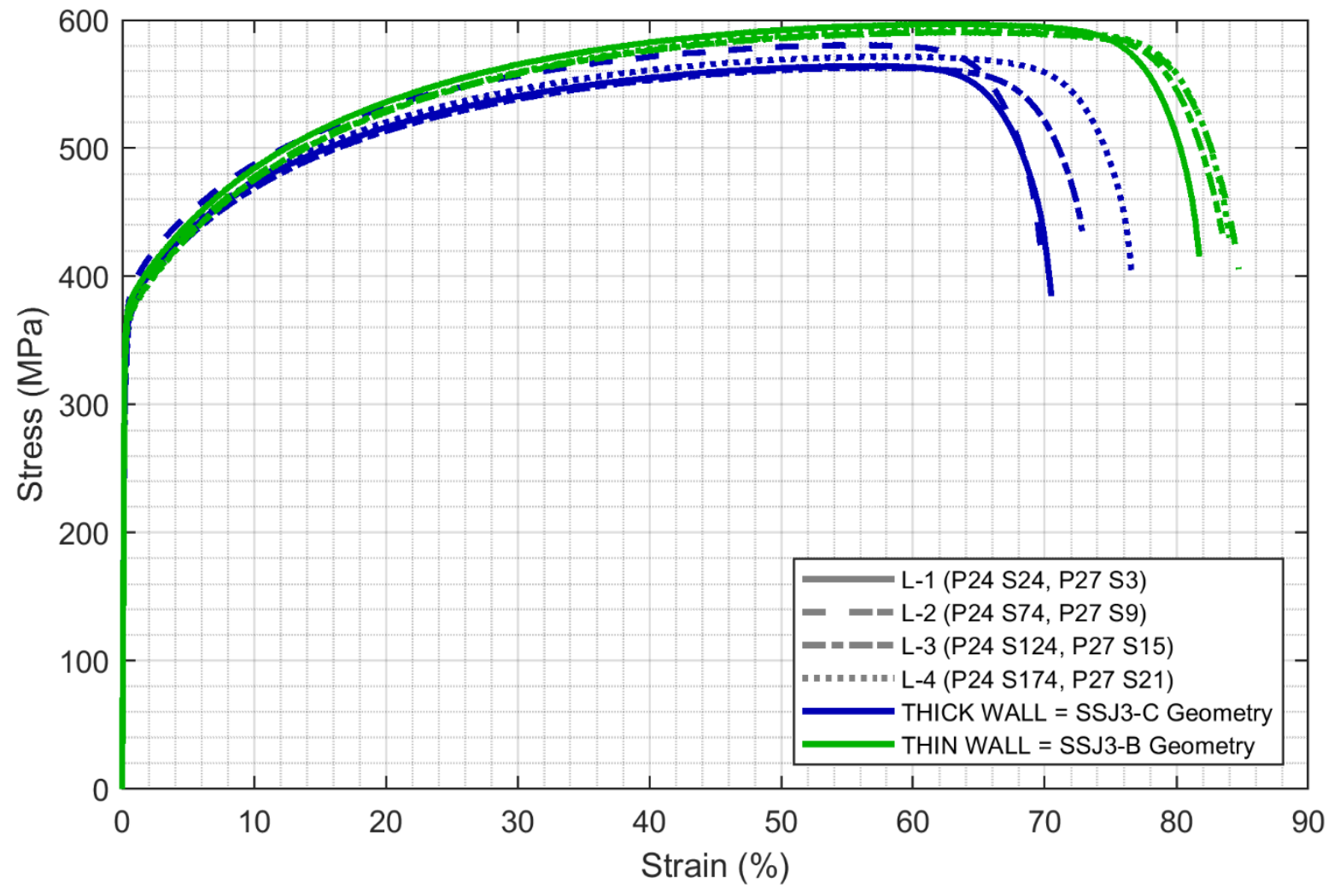

Figure 31. Selected stress-strain curves for "TCR Phase 1 Build 2" P24 and P27. Both parts were printed using the BEST processing parameter set. P24 is nominally $5 \mathrm{~mm}$ thick SSJ3-C geometry and P27 is a nominally $1.5 \mathrm{~mm}$ thick SSJ3-B geometry.

Although the AIR testing campaign yielded an extremely large amount of data, additional testingparticularly of samples from "TCR Phase 1 Build 4" and "TCR Phase 1 Build 5"-is required to understand the effects of all the key manufacturing process parameters identified by TCR. Additionally, targeted characterization efforts would allow the TCR team to understand many of the physical root causes of the observed variations in tensile properties and increase confidence in any AIR property predictions. Although beyond the scope of the TCR program, future applications of AIR-type schemas must generalize to different testing conditions (e.g., room temperature testing vs. elevated temperatures) without requiring full-scale duplications of the testing campaign.

\section{PROPERTY PREDICTION}

\subsection{IN SITU ANOMALY DETECTION}

In situ process anomalies are detected by using the dynamic segmentation convolutional neural network (DSCNN) described in Scime et al. [3]. The DSCNN, as implemented in the Peregrine software tool, takes as input the two visible-light images of the Concept Laser M2 powder bed at each printed layer of a build. One image is captured after layer fusion, and the other is captured after powder spreading; both images are fused into an image stack for processing by the DSCNN. The final output of the DSCNN is a segmentation mask of the layer with each pixel (approximately $100 \mu \mathrm{m}$ in size) classified as nominally printed material, anomaly-free powder, or an anomaly class, such as those introduced in Figure 2. Figure 32 shows the data flow through the DSCNN as implemented in the Peregrine software tool, and a simplified version of this data flow is also visible in context at marker (A) in Figure 1. 


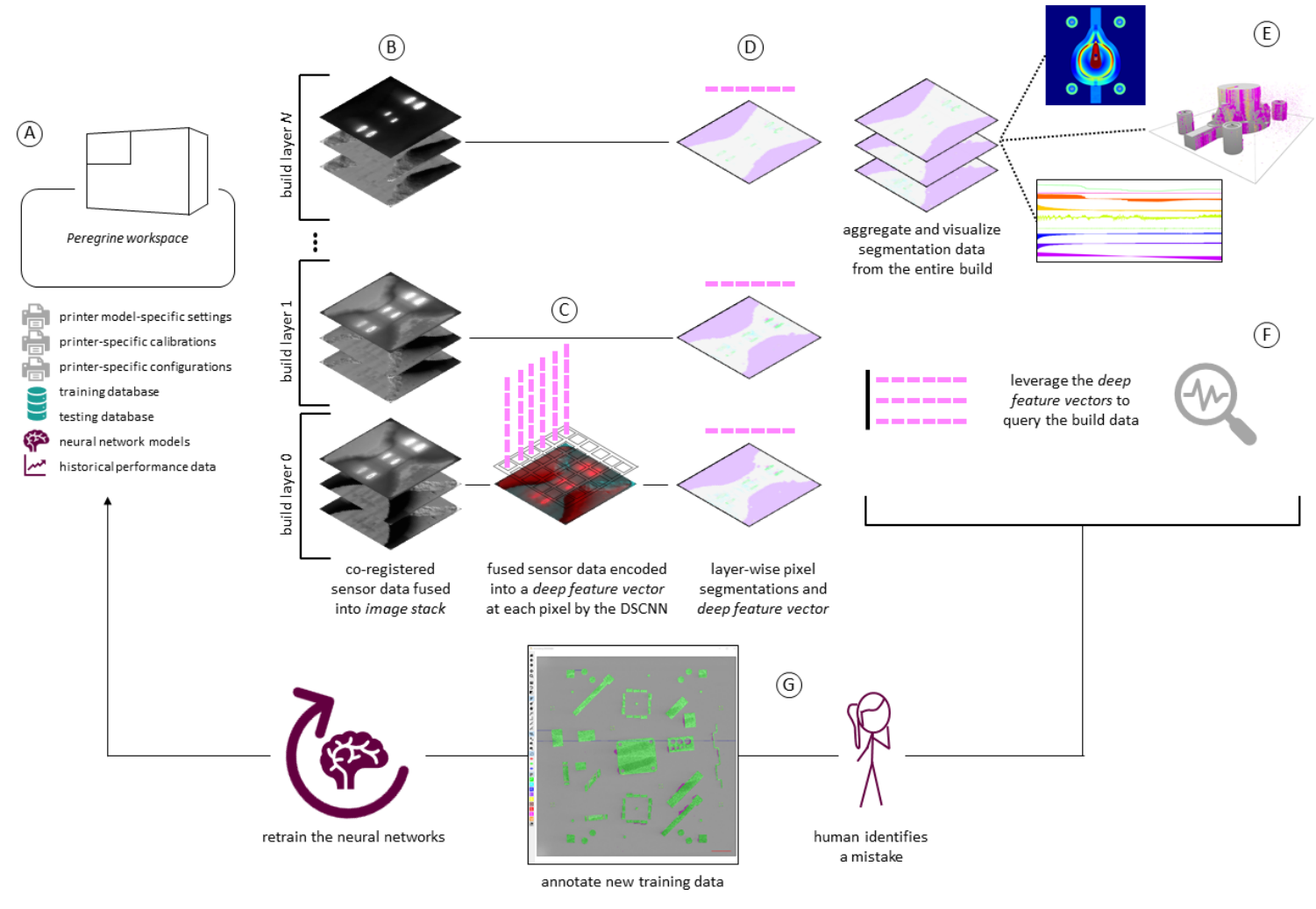

Figure 32. Peregrine's DSCNN data flow. (A) First, in situ image data are collected from the printer. (B) These data are coregistered with the CAD geometry and fused into an image stack. (C) The fused data are analyzed by the DSCNN, which outputs feature vectors at each pixel. (D) These feature vectors are used to produce a segmentation mask in which each pixel is assigned an L-PBF anomaly class. The layer-wise data for a given build are aggregated to enable (E) visualization and (F) data query capabilities. (G) Finally, any incorrect analysis results can be identified by a human, annotated, and fed back into the DSCNN to improve its performance. This figure and caption are similar to those submitted for publication in the Additive Manufacturing Journal in July 2021 [21].

Peregrine also parses the Concept Laser M2 machine log file produced at the end of each build. This log file records machine health and environmental sensor data as a time series throughout the build at data acquisition frequencies on the order of $1 \mathrm{~Hz}$. These log files are described in more detail in Scime et al. [22]; however, only a subset of the data available within the log file are leveraged by the AIR for predicting the tensile properties. Brief descriptions of the relevant sensor streams are provided in Table 7. 
Table 7. Description of the Concept Laser M2 log file values used by the AIR.

\begin{tabular}{l|l} 
Log file value & Description \\
\hline Layer time $(\mathrm{s})$ & The time required to melt the current build layer. \\
\hline Top flow rate $\left(\mathrm{m}^{3} / \mathrm{h}\right)$ & The argon gas flow rate measured at the top of the build chamber. \\
\hline Bottom flow rate $\left(\mathrm{m}^{3} / \mathrm{h}\right)$ & The argon gas flow rate measured at the bottom of the build chamber. \\
\hline Module Oxygen $(\%)$ & The oxygen concentration measured at the top of the build chamber. \\
\hline Build plate temperature $\left({ }^{\circ} \mathrm{C}\right)$ & The temperature measured at the base of the build plate. \\
\hline Bottom flow temperature $\left({ }^{\circ} \mathrm{C}\right)$ & $\begin{array}{l}\text { The temperature of the argon shield gas flowing across the bottom of } \\
\text { the build chamber. }\end{array}$ \\
\hline Top flow temperature $\left({ }^{\circ} \mathrm{C}\right)$ & $\begin{array}{l}\text { The temperature of the argon shield gas flowing across the top of the } \\
\text { build chamber. }\end{array}$ \\
\hline Bottom chamber temperature $\left({ }^{\circ} \mathrm{C}\right)$ & The temperature measured at the bottom of the build chamber. \\
\hline Top chamber temperature $\left({ }^{\circ} \mathrm{C}\right)$ & The temperature measured at the top of the build chamber. \\
\hline Build chamber position $(\mathrm{mm})$ & $\begin{array}{l}\text { The vertical position of the build chamber which serves as a proxy for } \\
\text { the height of the current build layer. }\end{array}$ \\
\hline Target ventilator flow rate $\left(\mathrm{m}^{3} / \mathrm{h}\right)$ & $\begin{array}{l}\text { The goal argon flow rate through the ventilator, as set by the printer } \\
\text { technician. }\end{array}$ \\
\hline Ventilator speed $(\%)$ & $\begin{array}{l}\text { The speed of the ventilator motor as a percentage of its maximum } \\
\text { speed. }\end{array}$
\end{tabular}

\subsection{SUPER-VOXEL FEATURE VECTORS}

After the L-PBF process anomalies are detected by the DSCNN, this information must be packaged into a feature vector that can be ingested by an ML model and used to predict the local tensile properties, as shown at marker (C) in Figure 1. Ideally, a DL algorithm would be used to automatically optimize the design and composition of this feature vector. Unfortunately, such an algorithm would require a prohibitively large number of tensile tests for training, as discussed in Section 1. Therefore, the AIR substitutes human domain expertise at this AI algorithm interface to engineer feature vectors, as depicted at marker (B) in Figure 1. Based on the AM community's understanding of the underlying L-PBF process physics, the following information is proposed to be important for predicting local tensile properties and thus is included in the feature vectors: (1) information about the DSCNN anomaly classifications and their distribution within a local area; (2) information about the local part geometry, specifically the distance of any point from the planar edge of the as-built part and the distance of any point from an overhanging surface in the vertical build direction; and (3) certain key machine health and environmental sensor data, as enumerated in Table 7 and including information regarding the melting time, layer height, shielding gas, environmental oxygen concentration, and environmental temperatures. The full list of 24 features is provided in Table 8. 
Table 8. Description of super-voxel features available to the AIR to predict local tensile properties.

\begin{tabular}{|c|c|}
\hline Feature name & Description \\
\hline Powder & $\begin{array}{l}\text { Represents the distribution of anomaly-free powder predicted by the } \\
\text { DSCNN within the super-voxel. }\end{array}$ \\
\hline Printed & $\begin{array}{l}\text { Represents the distribution of anomaly-free printed material predicted by the } \\
\text { DSCNN within the super-voxel. }\end{array}$ \\
\hline Recoater hopping & $\begin{array}{l}\text { Represents the distribution of recoater hopping anomalies predicted by the } \\
\text { DSCNN within the super-voxel. }\end{array}$ \\
\hline Recoater streaking & $\begin{array}{l}\text { Represents the distribution of recoater streaking anomalies predicted by the } \\
\text { DSCNN within the super-voxel. }\end{array}$ \\
\hline Incomplete spreading & $\begin{array}{l}\text { Represents the distribution of incomplete spreading anomalies predicted by } \\
\text { the DSCNN within the super-voxel. }\end{array}$ \\
\hline Swelling & $\begin{array}{l}\text { Represents the distribution of swelling anomalies predicted by the DSCNN } \\
\text { within the super-voxel. }\end{array}$ \\
\hline Debris & $\begin{array}{l}\text { Represents the distribution of debris anomalies predicted by the DSCNN } \\
\text { within the super-voxel. }\end{array}$ \\
\hline Super-elevation & $\begin{array}{l}\text { Represents the distribution of super-elevation anomalies predicted by the } \\
\text { DSCNN within the super-voxel. }\end{array}$ \\
\hline Soot & $\begin{array}{l}\text { Represents the distribution of soot anomalies predicted by the DSCNN } \\
\text { within the super-voxel. }\end{array}$ \\
\hline Misprint & $\begin{array}{l}\text { Represents the distribution of misprint anomalies predicted by the DSCNN } \\
\text { within the super-voxel. }\end{array}$ \\
\hline Overhang distance & $\begin{array}{l}\text { Encodes the average vertical distance of pixels within the super-voxel to the } \\
\text { nearest overhanging surface. }\end{array}$ \\
\hline Distance from part edges & $\begin{array}{l}\text { Encodes the average planar distance of pixels within the super-voxel to the } \\
\text { nearest as-built part edge. }\end{array}$ \\
\hline Layer time & The time required to melt the current build layer. \\
\hline Top flow rate & The argon gas flow rate measured at the top of the build chamber. \\
\hline Bottom flow rate & The argon gas flow rate measured at the bottom of the build chamber. \\
\hline Module Oxygen & The oxygen concentration measured at the top of the build chamber. \\
\hline Build plate temperature & The temperature measured at the base of the build plate. \\
\hline Bottom flow temperature & $\begin{array}{l}\text { The temperature of the argon shield gas flowing across the bottom of the } \\
\text { build chamber. }\end{array}$ \\
\hline Top flow temperature & $\begin{array}{l}\text { The temperature of the argon shield gas flowing across the top of the build } \\
\text { chamber. }\end{array}$ \\
\hline Bottom chamber temperature & The temperature measured at the bottom of the build chamber. \\
\hline Top chamber temperature & The temperature measured at the top of the build chamber. \\
\hline Build chamber position & $\begin{array}{l}\text { The vertical position of the build chamber which serves as a proxy for the } \\
\text { height of the current build layer. }\end{array}$ \\
\hline Target ventilator flow rate & $\begin{array}{l}\text { The goal argon flow rate through the ventilator, as set by the printer } \\
\text { technician. }\end{array}$ \\
\hline Ventilator speed & The speed of the ventilator motor as a percentage of its maximum speed. \\
\hline
\end{tabular}

To construct the feature vectors, the DSCNN anomaly segmentation masks for a given build layer are first convolved with a truncated Gaussian kernel (Figure 33), which effectively "blurs" the data and results in a vector of numbers at each $100 \mu \mathrm{m}$ pixel. Each vector represents one measure of the distribution of anomalies located in the region surrounding each pixel. The blurring is also useful as the registration between the in situ image data and, therefore, the DSCNN results, and the measured tensile properties from the SS-J3 gauge sections are expected to have errors on the order of several hundred microns. Next, a standard distance transform is performed to measure the planar distance of each pixel to the nearest asbuilt part edge; a similar procedure is performed in the vertical build direction to measure the distance from each pixel to the nearest overhanging surface. Finally, the log file data for the current layer are assigned to each pixel in the given layer. 

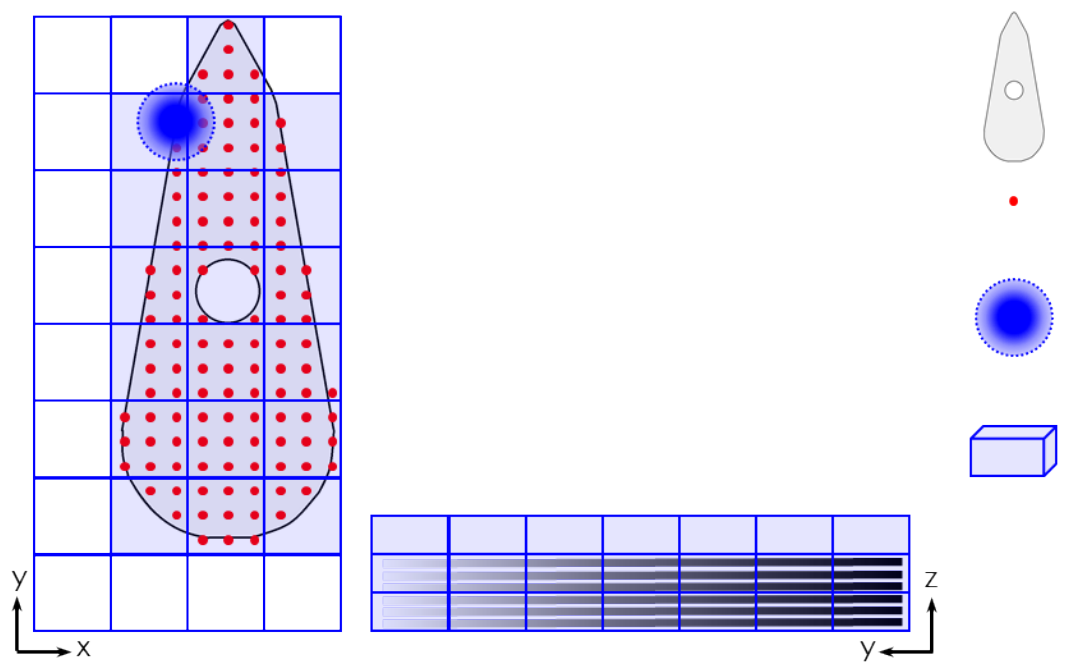

CAD-defined

part geometry

a single pixel lying

on top of a part

a truncated Gaussian

averaging filter with $\sigma=\frac{v_{x y}}{2}$

a super voxel which averages all valid $(\bullet)$ values in a planar area $v_{x y} \times v_{x y}$ and vertically through the number of layers corresponding to $v_{z}$

Figure 33. A graphical representation of the super-voxelization process. $\sigma$ is the standard deviation of the Gaussian kernel, $v_{x y}$ is the planar super-voxel resolution, and $v_{z}$ is the vertical resolution.

At this stage in the algorithm, feature vectors have been calculated at a planar resolution of approximately $100 \mu \mathrm{m} \times 100 \mu \mathrm{m}$ and a vertical resolution equal to the layer thickness of $50 \mu \mathrm{m}$. This results in a significant resolution mismatch between the in situ data and the tensile property data that is resolved only to the size of the SS-J3 gauge sections, which is $1,200 \times 750 \mu \mathrm{m}$ in-plane and 5,000 $\mu \mathrm{m}$ vertically. Therefore, the feature vectors must be averaged into super-voxels, which are sized at $1,000 \mu \mathrm{m} \times 1,000$ $\mu \mathrm{m}$ in-plane and 3,500 $\mu \mathrm{m}$ vertically. The super-voxels are defined on a fixed grid, as shown in Figure 33. To mitigate edge effects, in-plane averaging is only applied to pixels that lie on the part while the vertical averaging is performed with a moving box filter centered on the current build layer. Critically, supervoxelization also dramatically reduces the computer memory requirements associated with processing the feature vectors. The result is one feature vector at each super-voxel composed of the features enumerated in Table 8. Figure 34 shows several examples of super-voxelized features from the "TCR Phase 1 Build 2" build. 

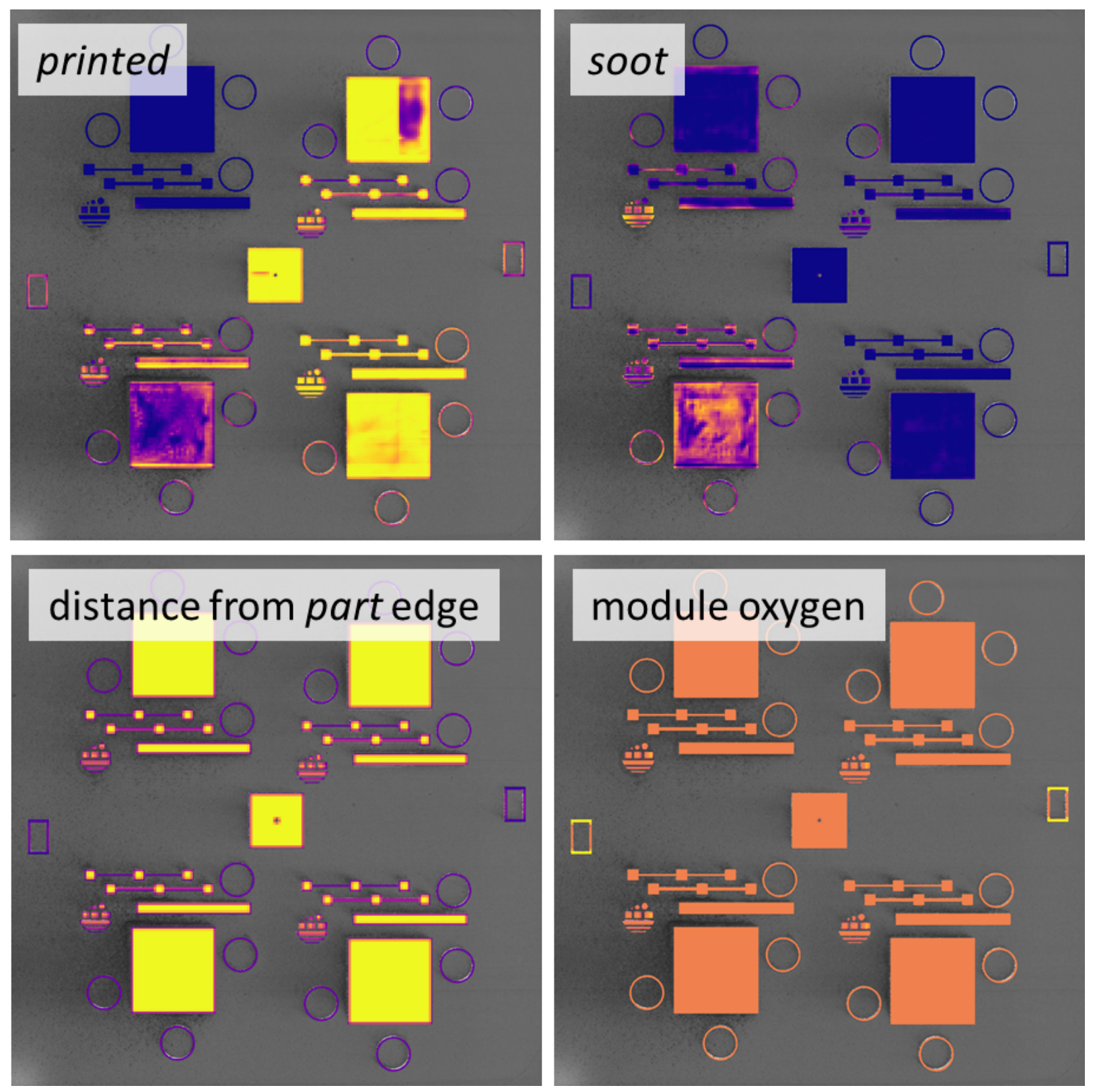

Figure 34. Example super-voxel features from the "TCR Phase 1 Build 2" build overlaid on top of the corresponding in situ image of the powder bed.

The "printed" feature encodes information about the distribution of anomaly-free printed material, as predicted by the DSCNN. The "soot" feature encodes information about the distribution soot anomalies, as predicted by the DSCNN. The "distance from

part edge" feature encodes information about the distance of the pixels within the super-voxel from the planar part edge. The "module oxygen" feature encodes information about the oxygen concentration in the build chamber at the layers represented by the super-voxel. The absolute values of all the features in this figure were normalized across the entire build. 


\subsection{LOCAL PROPERTY PREDICTION MODELS}

After all Phase 1 tensile data are collected, the AIR will be trained to predict UTS, YTS, UE, and TE; however, only prediction of UTS values is addressed in this report. UTS was selected because it is a relevant property for predicting burst tube failure, which will be used in FY22 to validate the AIR, as shown at marker (D) in Figure 1. Three different ML prediction models were explored, all of which take as input the feature vectors described in Section 4.2:

1. support vector machine (SVM) [23],

2. linear regression model (LRM) [23], and

3. generalized neural network (GNN) [24].

To facilitate measuring the relative performance of the various models and enable the SVM binary classifier, a UTS threshold of $485 \mathrm{MPa}$ was chosen based on ASTM A240 [25] and ASTM A666 [26]. UTS values above the threshold are considered "good," and UTS values below the threshold are considered "bad." For all models, the ground truth tensile data and their associated super-voxel-feature vectors were split into training, validation, and test sets. The available features (Table 8) were evaluated by using association rules to identify the features that have the highest variation and association with other high variance features. Among an initial set of available training data sourced exclusively from "TCR Phase 1 Build 2," four features were identified as important through the association rules: \{powder, debris, incomplete spreading, soot\}. The SVM that used these features had high accuracy, precision, and recall scores. However, when this same subset of features was used in the LRM, it resulted in a large uncertainty so that super-voxels with similar feature vectors could be predicted to have UTS values between $370 \mathrm{MPa}$ and $550 \mathrm{MPa}$, despite the model showing a decent coefficient of determination $\left(R^{2}\right.$ value).

Once the remainder of the training data from "TCR Phase 1 Build 2" and "TCR Phase 1 Build 3" were included, the SVM maintained high classification accuracy, precision, and recall scores. However, the LRM performed substantially worse relative to the reduced dataset. The four identified features appear to not fully account for the variance observed in the ground truths, and as a result, the predictions continued to show high uncertainties. The association rules algorithm was again applied to the complete set of super-voxels to remove any linearly dependent features and outlier features that did not correlate to outlier ground truth values. Outliers were identified by using statistical outlier detection methods and then correlated to the ground truth values and other features to determine whether the ground truth value for that observation was an outlier.

At this stage, the expanded set of features of importance was \{powder, printed, recoater hopping, recoater streaking, incomplete spreading, swelling, debris, super-elevation, soot, misprint, overhang distance\}. Only the overhang distance feature values were normalized over the training dataset. This vector of eleven features was used with the SVM with results similar to the SVM model using only the four initially identified features. The LRM using the expanded feature set showed a slight improvement in the coefficient of determination but hinted at a nonlinear relationship between input features and the target prediction. The nonlinear relationship is indicated by the sharp cutoff bounds of the upper and lower predicted values across the range of target values as shown in Figure 36 in the following section. To investigate a nonlinear relationship between input features and a target, a GNN architecture based on Behler et al. [24] was implemented. The basic architecture provided increased agreement between the predicted and target values, but uncertainty in the predicted UTS values remained above $40 \mathrm{MPa}$. Therefore, modifications to the GNN architecture (Figure 35) were made to further improve the coefficient of determination. 


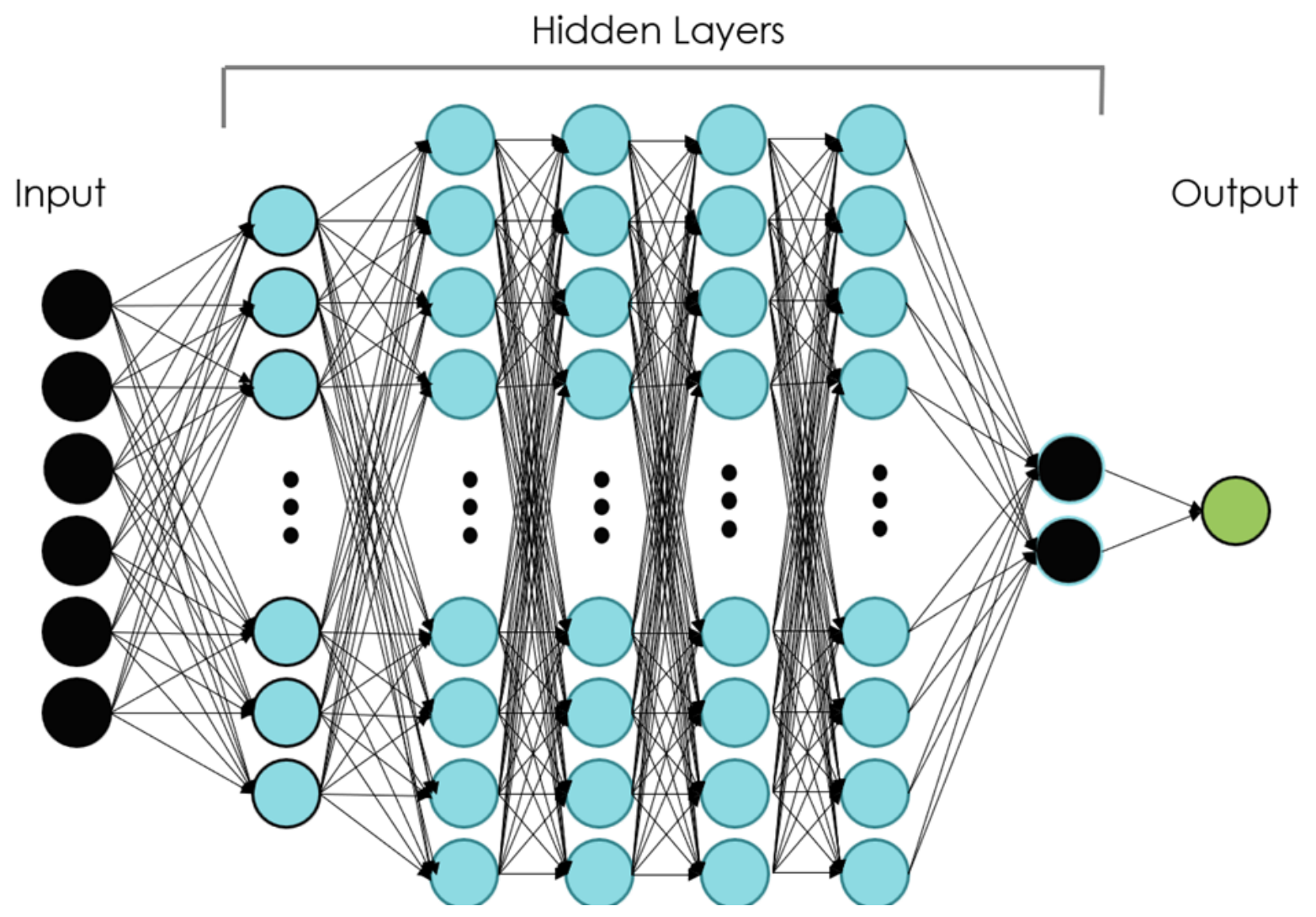

Figure 35. Representation of the modified GNN with five hidden layers consisting of 32, 128, 128, 128, 128, and 2 nodes, respectively, and an output layer. Each hidden layer uses a ReLU activation function, and the output layer uses a linear activation function. The node in the output layer predicts the local UTS value of the super-voxel.

\subsection{PRELIMINARY RESULTS}

As described in Section 4.3, the ML models evolved from a binary classification algorithm that uses four input features to a full GNN that uses 11 input features to predict the UTS of each super-voxel. The performances of the SVM models are shown in Table 9 and Table 10. Both models had high accuracy, precision, and recall for each classification. Additionally, the accuracy rating for both models is higher than the true positive rate of $82 \%$ for the test set.

Table 9. Confusion matrix for the SVM that uses a four-feature input vector.

\begin{tabular}{l|c|c|c|c} 
Classification & Precision & Recall & F1 Score & Observations \\
\hline$<485 \mathrm{MPa}$ & 0.92 & 0.80 & 0.85 & 123 \\
$>485 \mathrm{MPa}$ & 0.96 & 0.98 & 0.97 & 564 \\
Accuracy & 0.95 & & &
\end{tabular}

Table 10. Confusion matrix for the SVM that uses an 11 feature input vector.

\begin{tabular}{l|c|c|c|c} 
Classification & Precision & Recall & F1 Score & Observations \\
\hline$<485 \mathrm{MPa}$ & 0.90 & 0.84 & 0.87 & 123 \\
$>485 \mathrm{MPa}$ & 0.97 & 0.98 & 0.97 & 564 \\
Accuracy & 0.95 & & &
\end{tabular}


The LRM that uses four input features shows a poor coefficient of determination of 0.649 and has an average uncertainty of $53.8 \mathrm{MPa}$. Additionally, the LRM fails to predict UTS values above $575 \mathrm{MPa}$ or below $363 \mathrm{MPa}$. The LRM performance is shown in Figure 36 with the ground truth UTS values reported on the $x$-axis and the AIR predictions reported on the $y$-axis. The upper right quadrant of Figure 36 encompasses the super-voxels with UTS values predicted to be higher than $485 \mathrm{MPa}$ that also have a ground truth UTS value greater than $485 \mathrm{MPa}$. To a first order, these are "good" super-voxels that were correctly predicted by the AIR to be "good." The lower right quadrant encompasses "good" super-voxels that were incorrectly predicted to be "bad." The lower left quadrant encompasses super-voxels that are "bad" and have been correctly predicted by the AIR to be "bad." Finally, the upper left quadrant encompasses super-voxels that are "bad" but were incorrectly predicted to have "good" tensile properties. From a regulatory perspective, this is the most critical set of misclassifications to reduce with other model topologies.

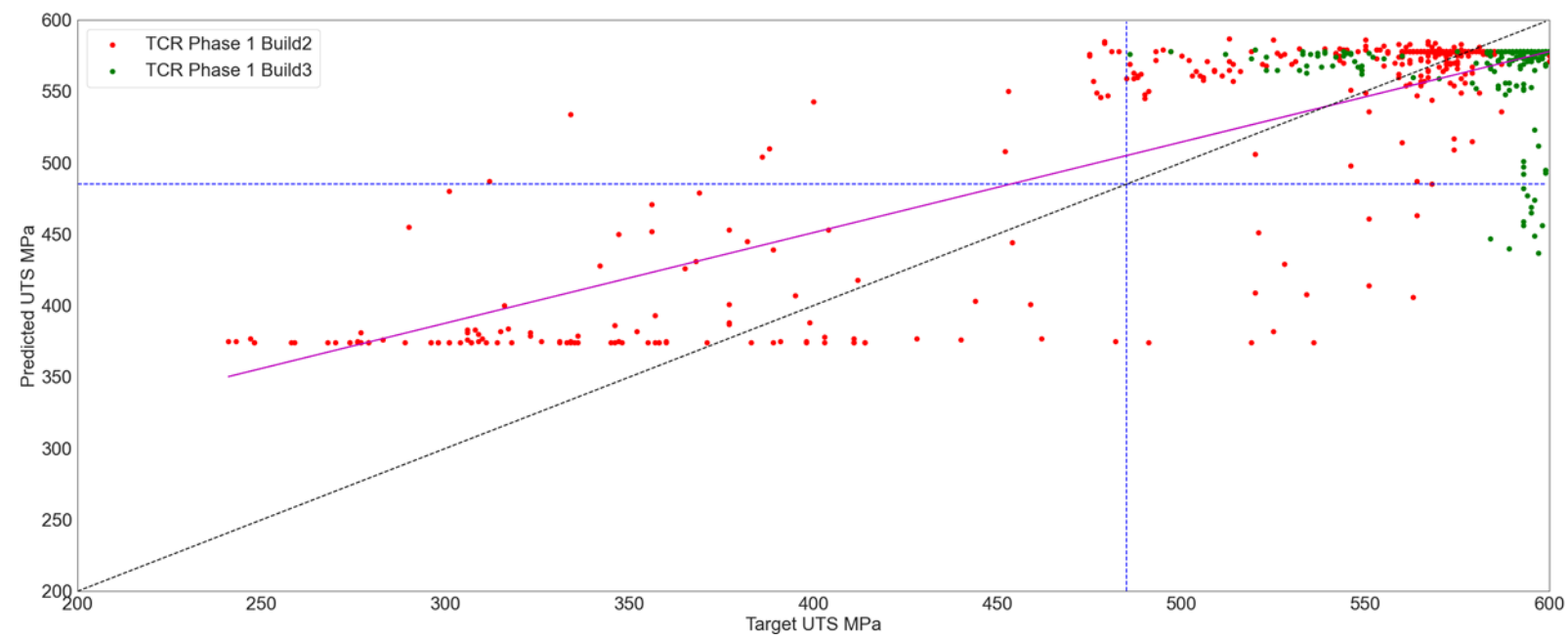

Figure 36. Predicted vs. ground truth UTS values for the four-feature LRM. The plot is divided into quadrants based on predicted and target values at the $485 \mathrm{MPa}$ threshold. Each datapoint represents a super-voxel, and each SS-J3 sample gauge section could contain multiple super-voxels. If the model performed perfectly, then all datapoints would be along the dotted black line. The solid magenta line is a linear trendline fit through the datapoints.

As shown in Figure 37, retraining the LRM using the 11 element feature vector improved the upper UTS predictions for "TCR Phase 1 Build 3" but still resulted in a hard cutoff bound for samples printed in "TCR Phase 1 Build 2." The coefficient of determination remained poor at only 0.656. This behavior suggests a nonlinear correlation between the features and target UTS values. The coefficient of determination changed slightly in the positive direction because of the higher UTS predictions for samples from "TCR Phase 1 Build 3" and slightly lower prediction uncertainty of 53.3 MPa. 


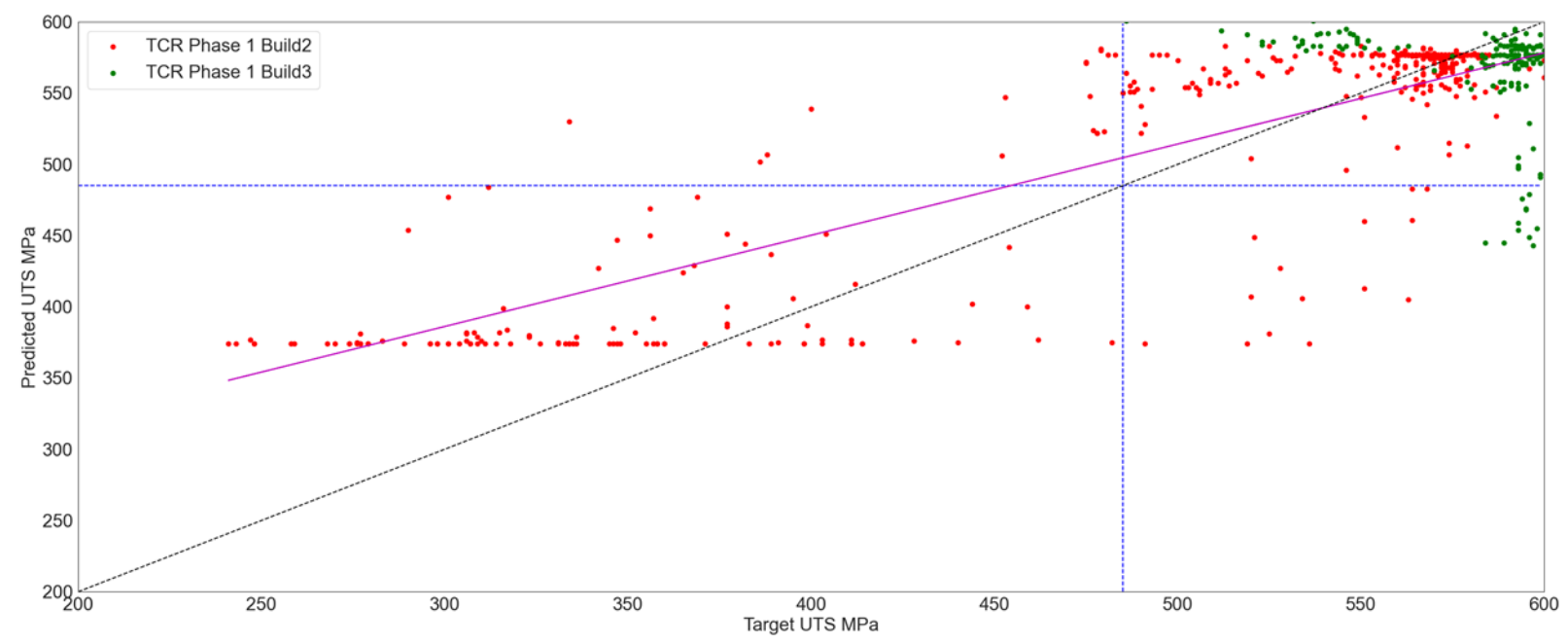

Figure 37. Predicted vs. ground truth UTS values for the 11 feature LRM. The plot is divided into quadrants based on predicted and target values at the $485 \mathrm{MPa}$ threshold. Each datapoint represents a super-voxel, and each SS-J3 sample gauge section could contain multiple super-voxels. If the model performed perfectly, then all datapoints would be along the dotted black line. The solid magenta line is a linear trendline fit through the datapoints.

Figure 38 shows the GNN predictions based on the 11 element feature vectors. As anticipated, the GNN can learn the nonlinear relationships between the features and the target values better than the LRM. The GNN coefficient of determination was the highest of all three regression models at 0.877 . This model reduced the uncertainty of the predictions to $31.9 \mathrm{MPa}$ and did not predict any "good" super-voxels from "TCR Phase 1 Build 3" below the threshold UTS value. Importantly, there is also an appreciable reduction in the number of "bad" super-voxels with predicted UTS values above the $485 \mathrm{MPa}$ threshold. The upper and lower bounds of the model predictions also changed with the upper bound showing a tighter correspondence to the target values. The low-end prediction bound heavily influences the coefficient of determination for the GNN. Overall, $96.2 \%$ of the 687 super-voxels in the test set were correctly predicted by the AIR as either above or below the $485 \mathrm{MPa}$ threshold. Further refinements of the GNN architecture and its predictions will require testing additional SS-J3 samples and gaining an understanding of the underlying mechanisms that result in the apparent outlying UTS predictions. 


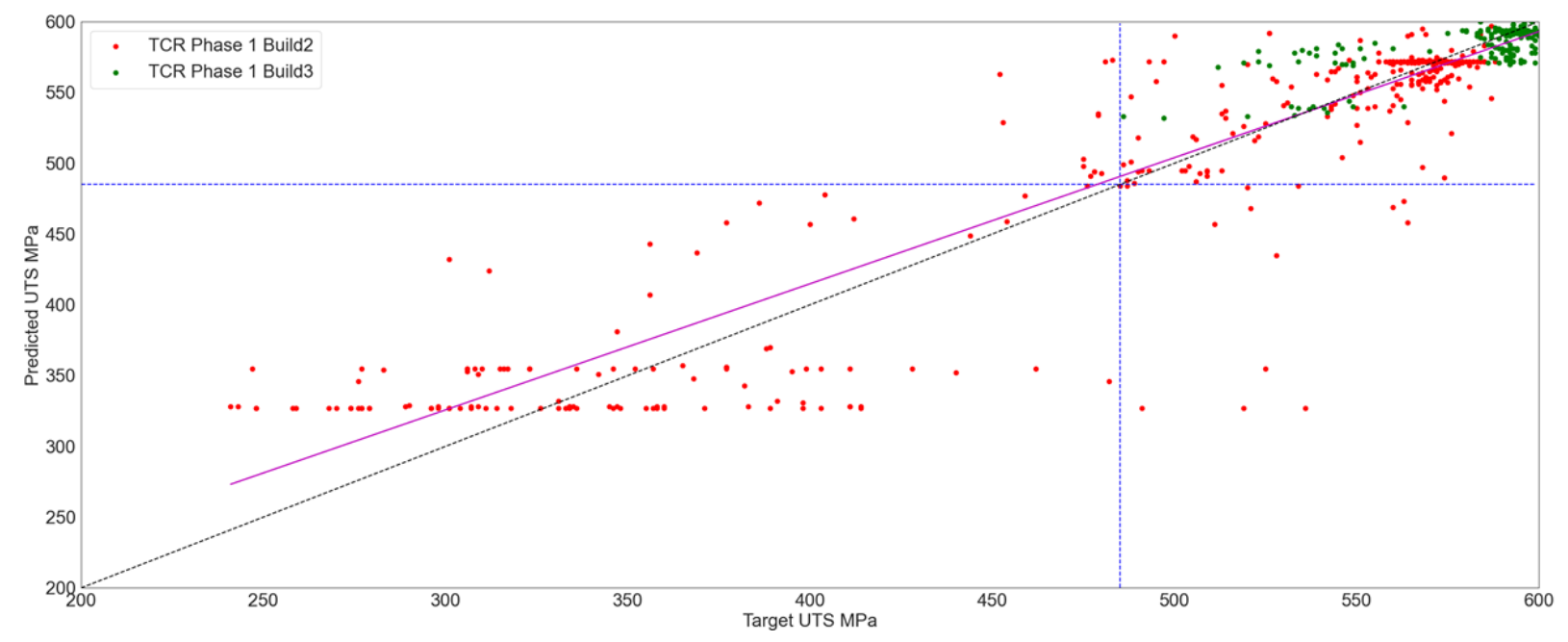

Figure 38. Predicted vs. ground truth UTS values for the 11 feature GNN. The plot is divided into quadrants based on predicted and target values at the $485 \mathrm{MPa}$ threshold. Each datapoint represents a super-voxel, and each

SS-J3 sample gauge section could contain multiple super-voxels. If the model performed perfectly, then all datapoints would be along the dotted black line. The solid magenta line is a linear trendline

fit through the datapoints.

\subsection{DEMONSTRATION OF THE AIR PIPELINE}

Once an optimal property prediction ML algorithm is selected, it will be integrated into the Peregrine software tool to provide property predictions for arbitrary part geometries. In FY21, the common interfaces between a property prediction algorithm and Peregrine were defined, and the full AIR pipeline was demonstrated with a shallow neural network known as a perceptron. For this demonstration, the perceptron was trained to predict local UTS using an early batch of UTS ground truth data, and then the trained perceptron was used to analyze the "TCR Phase 1 Build 2" build. Figure 39a and Figure 39b show the preliminary UTS predictions for one layer of this build overlaid on the corresponding in situ powder bed image. UTS predictions are only available for super-voxels near the as-built part edges because the training data only included data from the SSJ3-A and SSJ3-B thin wall geometries and because the Peregrine pipeline outputs a value of "cannot predict" for super-voxels with feature vectors dissimilar to those observed during training. That is, this algorithm design decision seeks to ensure that extrapolated values are not presented to Peregrine users. The predicted values can be compared with the ground truth data reported in Figure 39c. Although these results are highly preliminary, they demonstrate the feasibility of the highly novel AIR pipeline. 

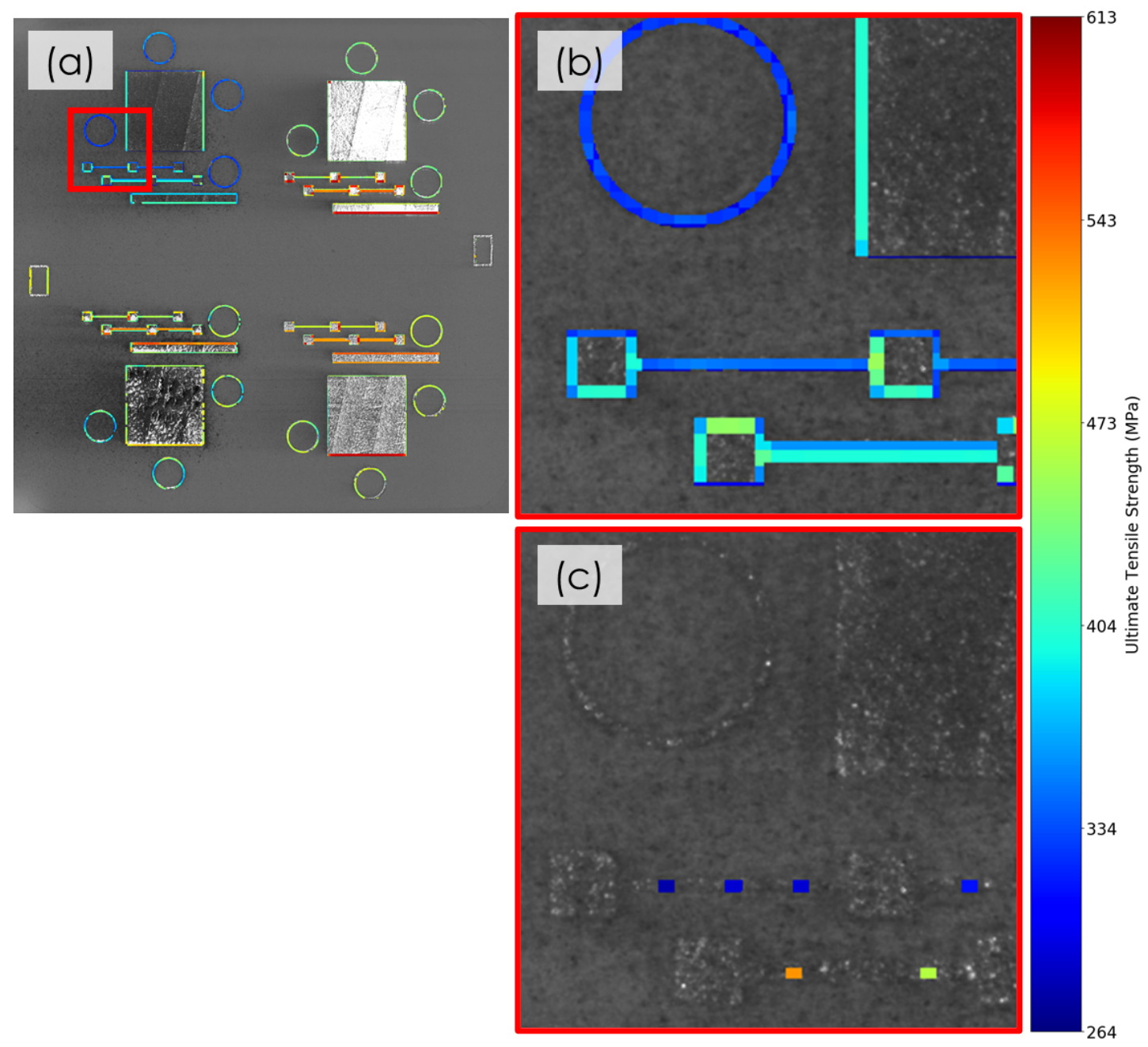

Figure 39. (a) Preliminary UTS predictions at each super-voxel for the as-built parts in the "TCR Phase 1 Build 2" build overlaid on top of the corresponding in situ image of the powder bed. Only values for supervoxels near the edges of the parts were predicted by Peregrine. (b) A zoomed-in region of (a) corresponding to the red detail box. (c) Ground truth UTS data for the same layer, where each super-voxel corresponds to the gauge section of an SS-J3 sample.

\section{DISCUSSION AND NEXT STEPS}

In FY21, the TCR team successfully demonstrated the AIR pipeline and its associated digital workflow. Overall, approximately 3,000 SS-J3 tensile samples were tested, which-although short of the FY21 target of 5,000 samples - represents an extremely large set of tensile property data by traditional standards. The prototype AIR is currently able to predict localized UTS values with a coefficient of determination ( $R^{2}$ value) of 0.877 . Importantly, if the AIR is used to determine whether a printed component clears a predefined safety threshold, its performance is even more promising with the final GNN implementation correctly classifying $96.2 \%$ of the test data as above or below a UTS threshold of $485 \mathrm{MPa}$. It is expected that the current AIR can be expanded to the remaining tensile properties-YTS, UE, and TE - with minimal additional effort. Note that these predictive capabilities are preliminary and are not expected to be fully generalizable until at least the remaining 2,000 SS-J3 samples from Phase 1 of the test campaign are tested. Validation of the AIR's ability to generalize will require testing the burst tubes in FY22. 
Significant programmatic delays and reprioritizations resulted in the loss of approximately 6 months of manufacturing and tensile testing time. As a result, only the five Phase 1 builds of the testing campaign were printed, and only a fraction of the targeted tensile samples was tested. Phase 2 will consist of another five builds and target the testing of an additional 5,000 tensile samples. Phase 2 builds will be designed after a full review of the Phase 1 data is complete so that specific gaps in the AIR training data can be addressed. Because of personnel limitations, the AIR does not currently incorporate feedstock or printer maintenance information into the feature vectors; these activities are now targeted for incorporation into the DP in FY22 under the MDF's Annual Operating Plan.

Significant room for optimization also remains for the AI algorithms that comprise the AIR. First, the DSCNN results should be improved, particularly in regard to recoater streaking and super-elevation anomaly detections, and this work has already begun. Once tensile data are collected from all five Phase 1 builds, the GNN model and the super-voxel input features can be optimized to improve property prediction performance and generalizability. Crucially, the novel AIR approach must also be validated by using both the burst tube tests described in Section 2 and by leveraging traditional material characterization techniques to understand the microstructural "root causes" behind the observed and predicted tensile properties. Although a lower priority for TCR, understanding how and if an AIR trained on a large quantity of room temperature tensile test data can be transferred to predicting other material properties (e.g., tensile properties after long-term thermal aging or irradiation) without requiring a similarly large number of tests will ultimately be necessary to ensure that the AIR approach is scalable. Finally, new visualization and analytics tools must be developed and incorporated into Peregrine to enable the domain experts and TCR engineers to fully leverage these powerful new property prediction capabilities.

\section{CONCLUSION}

The primary objective of the TCR program is to create a paradigm shift in the manufacturing of nuclear components and ultimately nuclear facilities by using the manufacturing flexibility provided by modern additive technologies. Toward this goal, the DP was designed to address the requirements for the certification and qualification of each additively manufactured component by capturing, organizing, and analyzing data collected throughout the entire manufacturing chain of each component. The authors' hypothesis is that this digital package contains more information than what is currently collected in traditional manufacturing settings and thus would provide a greater understanding of the quality and expected performance of the as-built components. During the first 2 years of the TCR program, the team has demonstrated the value of using in situ monitoring to enable the real-time detection of spatiotemporal features and defects in manufactured components. This year, the team conducted a methodical data collection campaign to link manufacturing strategies, conditions, and outcomes coupled with extensive mechanical testing to predict the performance of the components by using solely in situ process monitoring data. This report provides a detailed description of the experimental design, the data collection methodology, and the AI techniques used to provide convincing preliminary results on material properties and performance predictions. This work confirms the team's hypothesis and provides a strategy for designing experiments to expand this work for new materials and manufacturing conditions. The team also envisions cases in which the same strategy will be used to predict the performance of the component by using other performance metrics that depend on the actual intended use of the component.

Additionally, the team hopes that a broader adoption of this data collection campaign technique will lead the scientific community on a path to a collective goal of creating a manufacturing science database that can be leveraged for nuclear applications and other important US manufacturing objectives. 


\section{REFERENCES}

[1] ASTM. ISO/ASTM 52900: Standard Terminology for Additive Manufacturing 2015.

[2] Scime L, Singh A, Paquit V. A scalable digital platform for the use of digital twins in additive manufacturing. Manuf Lett 2021. https://doi.org/10.1016/j.mfglet.2021.05.007.

[3] Scime L, Siddel D, Baird S, Paquit V. Layer-wise anomaly detection and classification for powder bed additive manufacturing processes: A machine-agnostic algorithm for real-time pixel-wise semantic segmentation. Addit Manuf 2020;36:101453.

https://doi.org/10.1016/j.addma.2020.101453.

[4] Huning A, Fair R, Coates A, Paquit V, Scime L, Russell M, et al. Digital Platform Informed Certification of Components Derived from Advanced Manufacturing Technologies. Oak Ridge, TN (United States): 2021.

[5] Howard RH. S16-18- FUSSAM01: Generic Metal Irradiation Specimens. Oak Ridge, TN (United States): 2016.

[6] Scime L, Haley J, Halsey W, Singh A, Sprayberry M, Ziabari A, et al. Report on Progress of correlation of in-situ and ex-situ data and the use of artificial intelligence to predict defects. Oak Ridge, TN (United States): 2020. https://doi.org/10.2172/1684671.

[7] Russell M. TCR-AM-PLAN-002: TCR Digital Platform AM Fabrication and Test Plan. Oak Ridge, TN (United States): 2021.

[8] Russell M. TCR-AM-PLAN-003: TCR Digital Platform - Regulatory AM Fabrication and Burst Test Plan. Oak Ridge, TN (United States): 2021.

[9] Li Wei-Ying Chen M, Zhang X, Heidet F, Listwan EJ, Rink DL, Listwan JT. Location-Dependent Mechanical Property Evaluation on Additively Manufacture Materials. Argonne, Illinois: 2021.

[10] ASTM. ASTM E8/E8M: Standard Test Methods for Tension Testing of Metallic Materials 2016.

[11] ASTM. ASTM E4: Standard Practices for Force Verification of Testing Machines 2009.

[12] Callister WD, Rethwisch DG. Materials Science and Engineering: An Introduction. John Wiley \& Sons; 2010.

[13] Hertzberg RW. Deformation and Fracture Mechanics of Engineering Materials. Hoboken: John Wiley \& Sons; 1996.

[14] Kurzynowski T, Gruber K, Stopyra W, Kuznicka B, Chlebus E. Correlation between process parameters, microstructure and properties of $316 \mathrm{~L}$ stainless steel processed by selective laser melting. Mater Sci Eng A 2018;718:64-73.

[15] Field KG, Simpson J, Gussev MN, Wang H, Li M, Zhang X, et al. Handbook of advanced manufactured material properties from TCR structure builds at ORNL. Oak Ridge, TN (United States): 2019.

[16] Deev AA, Kuznetcov PA, Petrov SN. Anisotropy of mechanical properties and its correlation with the structure of the stainless steel 316L produced by the SLM method. Phys Procedia 2016;83:789-96.

[17] Im YD, Kim KH, Jung KH, Lee YK, Song KH. Anisotropic Mechanical Behavior of Additive Manufactured AISI 316L Steel. Metall Mater Trans A 2019;50A:2014-21.

[18] Charmi A, Falkenberg R, Avila L, Mohr G, Sommer K, Ulbricht A, et al. Mechanical anisotropy of additively manufactured stainless steel 316L: An experimental and numerical study. Mater Sci Eng A 2021;799:140-54.

[19] Ni X, Kong D, Wen Y, Zhang L, Wu W, He B, et al. Anisotropy in mechanical properties and corrosion resistance of $316 \mathrm{~L}$ stainless steel fabricated by selective laser melting. Int J Miner Metall Mater 2019;26:319-28.

[20] Wang X, Muniz-Lerma JA, Shandiz MA, Sanchez-Mata O, Brochu M. Crystallographicorientation-dependent tensile behaviors of stainless steel 316L fabricated by laser powder bed fusion. Mater Sci Eng A 2019;766. 
[21] Scime L, Joslin C, Goldsby D, Dehoff R, Richardson D, Siddel D, et al. Peregrine: multi-modal data visualization and artificial intelligence analytics for powder bed additive manufacturing. Addit Manuf n.d.

[22] Scime L, Halsey W, Haley J, Singh A, Sprayberry M, Ziabari A, et al. Development of Monitoring Techniques for Laser Powder Bed Additive Manufacturing of Metal Structures (Progress Report). Oak Ridge, TN (United States): 2020. https://doi.org/10.2172/1675044.

[23] Bonaccorso G. Mastering Machine Learning Algorithms: Expert techniques to implement popular machine learning algorithms and fine-tune your models. Packt Publishing; 2018.

[24] Behler MPJ. Generalized Neural-Network Representation of High-Dimensional Potential-Energy Sources. Am Phys Soc 2007;98.

[25] ASTM. ASTM A240/A240M-20a: Standard Specification for Chromium and Chromium-Nickel Stainless Steel Plate, Sheet, and Strip for Pressure Vessels and for General Applications 2020.

[26] ASTM. ASTM A666-15: Standard Specification for Annealed or Cold-Worked Austenitic Stainless Steel Sheet, Strip, Plate, and Flat Bar 2015. 